\title{
THE AIRY PHASE OF SHALLOW-FOCUS SUBMARINE EARTHQUAKES
}

\author{
By Frank Press, Maurice Ewing, and Ivan Tolstox*
}

\begin{abstract}
An ExTEnsion of the theoretical work of Lamb and Pekeris shows that a prominent phase (Airy phase) consisting of a group of waves of period 9-11 seconds, traveling across the ocean with a velocity of approximately 0.7 of the speed of sound in water, should be present on the seismograms of shallow-focus submarine earthquakes. This arrival corresponds to normal mode propagation at a stationary value of group velocity through the acoustic system consisting of the ocean and the rigid ocean bottom.

A phase fitting this description has been observed on the Milne-Shaw instruments at Bermuda for a series of Dominican Republic shocks. The Wenner seismographs at Huancayo recorded the Airy phase of a shock southwest of the Galapagos Islands.

The study of the Airy phase of a sufficient number of sea quakes would provide information concerning the nature of the ocean bottom since the latter affects both the periods and velocities of the Airy phase.
\end{abstract}

\section{INTRODUCTION}

A number of seismograms of earthquakes occurring at sea were examined and it was found that for a series of these a new phase could be identified consisting of a group of waves of period 8-10 seconds, traveling across the ocean with a velocity of $0.7-0.8$ of the speed of sound in water. It is believed that this arrival corresponds to normal mode propagation at a stationary value of group velocity through the acoustic system consisting of the ocean and the rigid ocean bottom.

Pekeris ${ }^{1}$ predicted and later identified this phase on seismograms of explosive sounds in shallow water obtained by Worzel and Ewing. ${ }^{2}$ In his theory of normal mode propagation in two liquid layers, Pekeris named this arrival the Airy phase and showed that its period depended on the depth of the water and the velocity and density contrasts between the water and the bottom. That periods associated with a stationary value of group velocity would predominate at long distances had been recognized earlier by Jeffreys ${ }^{3}$ and Stoneley. ${ }^{4}$

\footnotetext{
* Manuscript received for publication May 3, 1949.

The research reported in this document has been made possible through support and sponsorship extended by the Geophysical Research Directorate of the Cambridge Field Station, AMC, U. S. Air Force, under Contract W-28-099 ac-396. It is published for technical information only and does not represent recommendations or conclusions of the sponsoring agency.

I C. L. Pekeris, "Theory of Propagation of Explosive Sounds in Shallow Water," in "Propagation of Sound in the Ocean," Geol. Soc. Amer., Mem. No. 27 (1948).

'J. L. Worzel and Maurice Ewing, "Explosive Sounds in Shallow Water," in "Propagation of Sound in the Ocean," Geol. Soc. Amer., Mem. No. 27 (1948).

${ }^{3}$ Harold Jeffreys, "On Surface Waves of Earthquakes," Mon. Not. Roy. Astrom. Soc., Geophys. Suppl., $1: 282-293$ (1926).

"Robert Stoneley, "Surface Waves Associated with $20^{\circ}$ Discontinuity," Mon. Not. Roy. Astron. Soc., Geophys. Suppl., 4:39-43 (1937).
} 


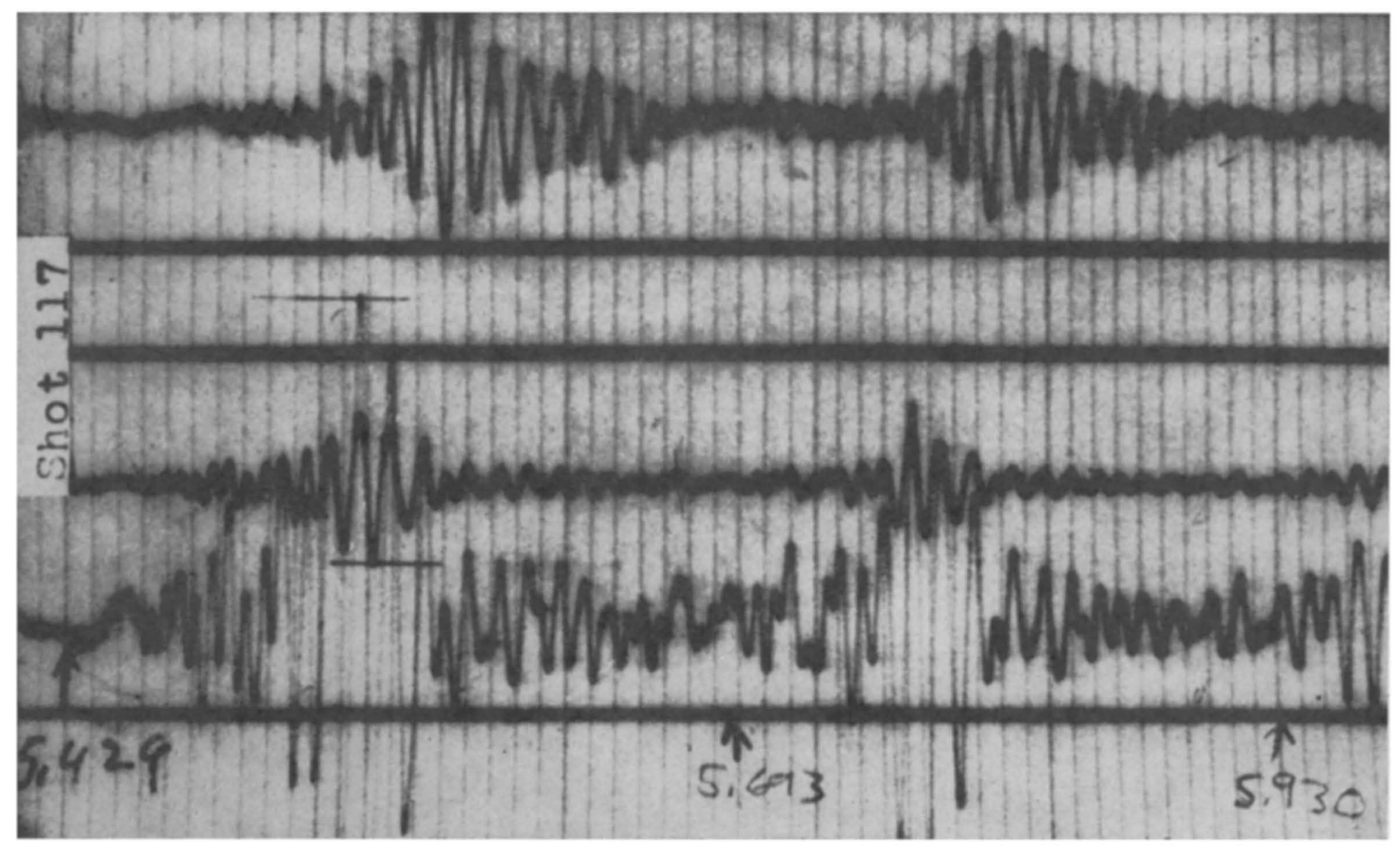

Fig. 1. Seismogram from a shallow-water shot illustrating the predominance of the Airy phase at large distances. The second arrival at about 5.7 seconds is due to the oscillation of the gas bubble. Trace 1 is from a geophone, and traces 4 and 5 are from a Naval Ordnance Laboratory Mark II sound-pressure hydrophone. 
A theory for the origin of microseisms in which microseism groups are considered to be Airy phases was advanced by Press and Ewing. ${ }^{5}$ This theory explains the mechanism of transmission of seismic energy from a storm at sea and correctly predicts that periods of three to nine seconds will predominate, the period being determined by the depth of water and the elastic constants of the bottom.

Whether the source of seismic energy is a disturbance at the ocean surface produced by a hurricane, an explosive charge detonated within the ocean, or an earthquake beneath the bottom, the normal mode theory indicates that a prominent Airy phase should be observed at a distant point. Figure 1, the seismogram from a shot of $5 \mathrm{lbs}$. in water 57 feet deep, at a distance of 4.5 nautical miles, illustrates the predominance of the Airy phase for shallow water explosions.

Although the treatment of the bottom as a liquid was adequate for the shallow water data to which Pekeris applied it, when one deals with submarine earthquakes in the deep ocean it becomes-necessary to consider the bottom as an elastic solid. In this paper, the Pekeris theory is extended to include the case of an impulsive point source of compressional waves within the bottom, which is taken as a solid.

\section{Propagation of Elaastic Waves in the Ocean from an Impulsive Point Source Beneath the Bottom}

The analagous problem of the generation of Rayleigh waves at the free surface of a semi-infinite solid for a source within the solid was studied by Lamb. ${ }^{6}$ Subsequent investigations by Stoneley ${ }^{7}$ and Sezawa ${ }^{8}$ dealt with the effect of the ocean on the transmission of seismic waves across the ocean bottom. Stoneley considered plane waves and calculated several values of phase and group velocity for long periods. Sezawa obtained an approximate solution for the propagation of cylindrical waves having great length as compared with the depth of the water. Lee ${ }^{9}$ calculated the amplitudes of Rayleigh waves for a system of two solids, where plane waves were transmitted through a superficial layer in contact with a semi-infinite medium, assuming various values for the elastic constants of the two media. Scholte ${ }^{10}$ gave a theory for the com-

\footnotetext{
${ }^{5}$ Frank Press and Maurice Ewing, "A Theory of Microseisms with Geological Applications," Trans. Amer. Geoph. Union, Vol. 29, No. 2, pp. 163-174 (1948)

6 Horace Lamb, "On the Propagation of Tremors over the Surface of an Elastic Solid," Phil. Trans., Roy. Soc. London, Ser. A, Vol. 203, pp. 1-42 (1904).

7 Robert Stoneley, "The Effect of the Ocean on Rayleigh Waves," Mon. Not. Roy. Astron. Soc., Geophys. Suppl., 1:349-356 (1926).

${ }^{8}$ Katsutada Sezawa, "On the Transmission of Seismic Waves on the Bottom Surface of an Ocean," Bull. Earthq. Res. Inst., Tokyo Imp. Univ., Vol. 9, Pt. 2, pp. 115-143 (1931).

${ }^{9}$ A. W. Lee, "Further Investigations of the Effect of Geological Structure upon Microseismic Disturbance," Mon. Not. Roy. Astron. Soc., Geophys. Suppl., 3:238-252 (1934).

${ }^{10} \mathrm{~J}$. G. Scholte, "Over Het Verband Tussen Zeegolven en Microseismen, I and II," Verslag Ned. Akad. Wet., 52:669-683 (1943).
} 
bined effects of gravity and compressibility in a layer of water in contact with an elastic solid bottom. Although Scholte's emphasis was not on propagation, he did prepare an excellent tabulation of phase velocities for the first mode. Press and Ewing ${ }^{11}$ presented complete curves of phase and group velocity for the first and second modes of motion in a liquid layer superposed on a semiinfinite solid bottom and pointed out the importance of stationary values of group velocity for elastic waves transmitted across the ocean.

In the present paper we make particular use of the methods and results of Lamb and Pekeris to derive the normal-mode solutions from which the wave motion at large distances from the source can be obtained.

Consider the propagation of elastic waves through the system consisting of a liquid layer of thickness $H$, density $\rho_{1}$, and compressional wave velocity $v_{1}$, superposed upon an infinitely thick, solid bottom of density $\rho_{2}$ whose compressional and distortional wave velocities are respectively $\alpha_{2}$ and $\beta_{2}$. $\lambda_{1}$ is the incompressibility of the liquid and $\lambda_{2}, \mu_{2}$ are Lamé's constants for the bottom, and are related to the elastic wave velocities as follows:

$$
\begin{aligned}
& v_{1}^{2}=\lambda_{1} / \rho_{1} \\
& \alpha_{2}^{2}=\left(\lambda_{2}+2 \mu_{2}\right) / \rho_{2} \\
& \beta_{2}^{2}=\mu_{2} / \rho_{2}
\end{aligned}
$$

The Cartesian coördinate system is chosen with the $x, y$ axes in the free surface and the $z$ axis vertically downward. Assuming symmetry about the $z$ axis, we have $r=\sqrt{x^{2}+y^{2}}$ and denote the displacements perpendicular and parallel to that axis by $q$ and $w$ respectively. The subscripts 1 and 2 refer to the liquid layer and bottom respectively.

Simple harmonic compressional point source within bottom.-We wish to determine the component displacements $q$ and $w$ due to a point source of compressional waves at $r=0, z=d+H$. In particular we will seek the normal mode solutions which predominate at large distances from the source.

Following the procedure of Lamb, we assume simple harmonic motion $e^{i \omega t}$ and introduce the functions $\varphi(r, z)$ and $\psi(r, z)$ defined by the equations:

$$
\begin{aligned}
& q_{1}=\partial \varphi_{1} / \partial r \\
& w_{1}=\partial \varphi_{1} / \partial z \\
& q_{2}=\partial \varphi_{2} / \partial r+\partial^{2} \psi_{2} / \partial r \partial z \\
& w_{2}=\partial \varphi_{2} / \partial z+\partial^{2} \psi_{2} / \partial z^{2}+\left(\omega^{2} / \beta_{2}{ }^{2}\right) \psi_{2}
\end{aligned}
$$

${ }^{11}$ Frank Press and Maurice Ewing, loc. cit. 
The vertical stress $p_{z z}$ and the tangential stress $p_{z r}$ can be expressed in terms of $q, w, \varphi, \psi$, and the elastic constants as follows:

$$
\begin{aligned}
& p_{z z}=\lambda \nabla^{2} \varphi+2 \mu \partial w / \partial z \\
& p_{z \tau}=\mu(\partial q / \partial z+\partial w / \partial r)
\end{aligned}
$$

It is required that the functions $\varphi$ and $\psi$ satisfy the equations:

$$
\begin{aligned}
& \left(\nabla^{2}+\omega^{2} / v_{1}^{2}\right) \varphi_{1}=0 \\
& \left(\nabla^{2}+\omega^{2} / \alpha_{2}^{2}\right) \varphi_{2}=0 \\
& \left(\nabla^{2}+\omega^{2} / \beta_{2}^{2}\right) \psi_{2}=0
\end{aligned}
$$

where $\nabla^{2}=\partial^{2} / \partial r^{2}+\frac{1}{r} \partial / \partial r+\partial^{2} / \partial z^{2}$.

It is convenient at this point to give an auxiliary solution where an external vertical force with magnitude $Z J_{0}(k r)$ per unit area acts upon a thin stratum coinciding with the interface between the two media. The functions $\varphi$ and $\psi$ must satisfy the boundary conditions:

$$
\begin{aligned}
\left(p_{z z}\right)_{1} & =0 \text { at } z=0 \\
\left(p_{z z}\right)_{2}-\left(p_{z z}\right)_{1} & =Z J_{0}(k r) \text { at } z=H \\
\left(p_{z r}\right)_{2} & =0 \text { at } z=H \\
w_{1} & =w_{2} \text { at } z=H
\end{aligned}
$$

Typical solutions of (5), (6) and (7) are of the form:

$$
\begin{aligned}
& \varphi_{1}=A \sin (\xi z) J_{0}(k r) \\
& \varphi_{2}=B e^{-\eta z} J_{0}(k r) \\
& \psi_{2}=C e^{-\zeta z} J_{0}(k r),
\end{aligned}
$$

where the separation constants $\xi, \eta, \zeta$, obtained by substituting (12)-(14) in (5)-(7), are

$$
\begin{aligned}
& \xi^{2}=\omega^{2} / v_{1}{ }^{2}-l^{2} \\
& \eta^{2}=k^{2}-\omega^{2} / \alpha_{2}{ }^{2} \\
& \zeta^{2}=k^{2}-\omega^{2} / \beta_{2}{ }^{2}
\end{aligned}
$$


$\xi, \eta, \zeta$ being positive real or positive imaginary. The time factor $e^{i \omega t}$ is temporarily omitted. The boundary condition expressed by (8) has been satisfied by the choice of $\varphi_{1}$. If equations (12)-(14) are substituted in the boundary conditions given by (9)-(11), using (3)-(4), three equations for the constants $A, B, C$ are obtained. Solving for $A, B$, and $C$ we have after some reduction:

$$
\begin{aligned}
& A=\frac{Z}{\mu_{2}} \frac{\omega^{2}}{\beta_{2}^{2}} \frac{\eta}{\xi F(k) \cos (\xi H)} \\
& B=\frac{Z}{\mu_{2}} \frac{\left(2 k^{2}-\omega^{2} / \beta_{2}^{2}\right) e^{\eta H}}{F(k)} \\
& C=\frac{Z}{\mu_{2}} \frac{2 \eta e^{\zeta H}}{F(k)}
\end{aligned}
$$

where $F(k)$ is given by:

$$
F(k)=\frac{\rho_{1} \omega^{4} \eta}{\rho_{2} \beta_{2}{ }^{4} \xi} \tan (\xi H)-\left[4 k^{2} \eta \zeta-\left(2 k^{2}-\omega^{2} / \beta_{2}^{2}\right)^{2}\right]
$$

For a source of compressional waves beneath the interface at the point $r=0, z=H+d$, the simplest solution which gives a compressional wave traveling radially outward in an unlimited solid is

$$
\varphi_{2}=\frac{e^{-i \frac{i !}{\alpha_{2}}}}{R}, \quad \psi_{2}=0
$$

where $R=\sqrt{x^{2}+y^{2}+(z-H-d)^{2}}$ denotes the radial distance from the point source. If an image is placed at $r=0, z=H-d$, we obtain a solution for which the tangential stress at $z=H$ vanishes, namely,

$$
\begin{aligned}
& \varphi_{2}=\frac{e^{-i \frac{\omega}{\alpha_{2}} R}}{R}+\frac{e^{-i \frac{\omega}{\alpha_{2}} R^{\prime}}}{R^{\prime}} \\
& \psi_{2}=0
\end{aligned}
$$

where $R^{\prime}=\sqrt{x^{2}+y^{2}+\left(z-H+\bar{d}^{2}\right.}$. From the integral form of the Bessel function Lamb ${ }^{12}$ showed that the above expression for $\varphi_{2}$ is equivalent to

$$
\begin{gathered}
\varphi_{2}=\int_{0}^{\infty} \frac{e^{-\eta(z-H+d)}}{\eta} J_{0}(k r) k d k+\int_{0}^{\infty} \frac{e^{\eta(z-H-d)}}{\eta} J_{0}(k r) k d k \\
=2 \int_{0}^{\infty} \frac{\cosh [\eta(z-H)]}{\eta} e^{-\eta d} J_{0}(k r) k d k .
\end{gathered}
$$

${ }^{12}$ Horace Lamb, loc. cit. 
From (3) and (4) we can write for $z=H$

$$
\begin{aligned}
& p_{z r}=0 \\
& p_{z z}=2 \mu_{2} \int_{0}^{\infty} \frac{\left(2 k^{2}-\omega^{2} / \beta_{2}^{2}\right)}{\eta} e^{-\eta d} J_{0}(k r) k d k .
\end{aligned}
$$

Referring to (9), we note that the condition for continuity of normal stress at the interface will be satisfied by superposing on (20) the solution obtained from (16) and (12)-(14) by putting

$$
Z=-\frac{2 \mu_{2}\left(2 k^{2}-\omega^{2} / \beta_{2}^{2}\right)}{\eta} e^{-\eta d} k d k
$$

and integrating with respect to $k$ between the limits 0 and $\infty$. It can be verified from (2), (12)-(14), and (16) that the condition for continuity of $w$ across the interface will be satisfied. Thus we may write for the formal, steady state solution for a periodic compressional point source:

$$
\begin{aligned}
\varphi_{1}= & -2 \frac{\omega^{2}}{{\beta_{2}}^{2}} \int_{0}^{\infty} \frac{\left(2 k^{2}-\omega^{2} / \beta_{2}{ }^{2}\right) \sin (\xi z)}{\xi F(k) \cos (\xi H)} e^{-\eta d} J_{0}(k r) k d k \\
\varphi_{2}= & 2 \int_{0}^{\infty} \frac{\cosh [\eta(z-H)]}{\eta} e^{-\eta d} J_{0}(k r) k d k \\
& -2 \int_{0}^{\infty} \frac{\left(2 k^{2}-\omega^{2} / \beta_{2}\right)^{2}}{\eta F(k)} e^{-\eta d} e^{-\eta(z-H)} J_{0}(k r) k d k \\
\psi_{2}= & -4 \int_{0}^{\infty} \frac{\left(2 k^{2}-\omega^{2} / \beta_{2}{ }^{2}\right)}{F(k)} e^{-\eta d} e^{-\zeta(z-H)} J_{0}(k r) k d k
\end{aligned}
$$

From (2), (24), and (25) we may write for the displacements $q_{H}, w_{H}$ at the surface of the solid bottom $(z=H)$ :

$$
\begin{aligned}
& q_{H}=-2 \frac{\omega^{2}}{\beta_{2}{ }^{2}} \int_{0}^{\infty} \frac{k}{F(k)}\left[\frac{\rho_{1} \omega^{2}}{\rho_{2} \beta_{2}^{2}} \frac{\tan (\xi H)}{\xi}-2 \zeta\right] e^{-\eta d} J_{1}(k r) k d k \\
& w_{H}=-2 \frac{\omega^{2}}{\beta_{2}{ }^{2}} \int_{0}^{\infty} \frac{\left(2 k^{2}-\omega^{2} / \beta_{2}{ }^{2}\right)}{F(k)} e^{-\eta d} J_{0}(k r) k d k
\end{aligned}
$$

Integrals of this general type have been evaluated by Lamb, ${ }^{13}$ Nakano, ${ }^{14}$ Sezawa, ${ }^{15}$ and Pekeris. ${ }^{16}$ The procedure is to transform the path of integration

\footnotetext{
13 Horace Lamb, loc. cit.

14 H. Nakano, "On Rayleigh Wave," Jap. Journ. Astron. and Geophys., Vol. II, No. 5, pp. 233-326 (1925).

í Katsutada Sezawa, "Love-Waves Generated from a Source of a Certain Depth," Bull. Earth. Res. Inst., Tokyo Imp. Univ., Vol. 13, Pt. 1, pp. 1-17 (1935).

${ }^{16}$ C. L. Pekeris, loc. cit.
} 
to the complex $k$-plane. The solutions can then be expressed as the sum of the residues of the integrands and two integrals along branch lines corresponding to the branch points $k=\omega / \alpha_{2}$ and $k=\omega / \beta_{2}$. The residues, which diminish as $1 / \sqrt{r}$ give the normal mode solutions, whereas the branch line integrals diminish as $1 / r^{2}$, and become negligible for large $r$.

Following this procedure and neglecting for the while the contributions of the branch line integrals, we find for large values of $r$ :

$$
\begin{aligned}
& q_{H}=2 i \pi \frac{\omega^{2}}{\beta_{2}{ }^{2}} \sum_{n} \sqrt{\frac{2}{\pi k_{n} r}} \frac{k_{n}^{2}}{\frac{\partial F\left(k_{n}\right)}{\partial k}}\left[\frac{\rho_{1}}{\rho_{2}} \frac{\omega^{2}}{\beta_{2}^{2}} \frac{\tan \left(\xi_{n} H\right)}{\xi_{n}}-2 \zeta_{n}\right] e^{-\eta_{n} d} e^{i\left(\omega t-k_{n} r-\pi / 4\right)} \\
& w_{H}=2 \pi \frac{\omega^{2}}{\beta_{2}^{2}} \sum_{n} \sqrt{\frac{2}{\pi k_{n} r}} \frac{k_{n}}{\frac{\partial F\left(k_{n}\right)}{\partial k_{n}}}\left(2 k_{n}{ }^{2}-\omega^{2} / \beta_{2}{ }^{2}\right) e^{-\eta_{n} d} e^{i\left(\omega t-k_{n} r-\pi / 4\right)}
\end{aligned}
$$

The subscript $n$ indicates that the quantity is to be evaluated at $k=k_{n}$, where $k_{n}$ are the roots of the period equation:

$$
F(k)=0
$$

From the factor $e^{i\left(\omega t-k_{n} r-\pi / 4\right)}$ it can be seen that the phase velocity $c_{n}$ can be expressed by

$$
c_{n}=\omega / k_{n}
$$

and (28), (29) can be written in the form

$$
\begin{aligned}
& q_{H}=\frac{2 \pi}{H^{2}} \sqrt{\frac{2}{\pi r}} \sum_{n} \frac{1}{\sqrt{k_{n}}} Q\left(k_{n}\right) e^{-\eta_{n} d} e^{i\left(\omega t-k_{n} r+\pi / 4\right)} \\
& w_{H}=\frac{2 \pi}{H^{2}} \sqrt{\frac{2}{\pi r}} \sum_{n} \frac{1}{\sqrt{k_{n}}} W\left(k_{n}\right) e^{-\eta_{n} d} e^{i\left(\omega t-k_{n} r+\pi / 4\right)}
\end{aligned}
$$

where

$$
\begin{gathered}
Q\left(k_{n}\right)=\frac{\left(k_{n} H\right)^{2}}{R\left(k_{n}\right)} \frac{c_{n}^{2}}{{\beta_{2}}^{2}}\left[\frac{\rho_{1}}{\rho_{2}} \frac{c_{n}^{2}}{{\beta_{2}}^{2}} \sqrt{\sqrt{1-c_{n}^{2} / \alpha_{2}^{2}}} \tan \left(k_{n} H \sqrt{c_{n}^{2} / v_{1}^{2}-1}\right)\right. \\
\left.-2 \sqrt{1-c_{n}^{2} / \alpha_{2}^{2}} \sqrt{1-c_{n}^{2} / \beta_{2}^{2}}\right] \\
W\left(k_{n}\right)=\frac{\left(k_{n} H\right)^{2}}{R\left(k_{n}\right)} \frac{c_{n}^{2}}{{\beta_{2}{ }^{2}}^{2}}\left(2-c_{n}^{2} / \beta_{2}^{2}\right) \sqrt{1-c_{n}{ }^{2} / \alpha_{2}^{2}}
\end{gathered}
$$


and

$$
\begin{aligned}
& R\left(k_{n}\right)=\frac{\rho_{1} c_{n}{ }^{4}}{\rho_{2} \beta_{2}{ }^{4}}\left\{\left[1+\frac{\left(1-c_{n}{ }^{2} / \alpha_{2}{ }^{2}\right)}{\left(c_{n}{ }^{2} / v_{1}{ }^{2}-1\right)}\right] \tan \frac{\left(k_{n} H \sqrt{c_{n}^{2} / v_{1}^{2}-1}\right)}{\sqrt{c_{n}^{2} / v_{1}^{2}-1}}\right. \\
& \left.-\frac{\left(k_{n} H\right)\left(1-c_{n}^{2} / \alpha_{2}^{2}\right)}{\left(c_{n}^{2} / v_{1}^{2}-1\right)} \sec ^{2}\left(k_{n} H \sqrt{c_{n}^{2} / v_{1}^{2}-1}\right)\right\} \\
& -4\left\{\left(3-2 c_{n}{ }^{2} / \alpha_{2}^{2}\right) \sqrt{1-c_{n}^{2} / \beta_{2}^{2}}+\frac{\left(1-c_{n}^{2} / \alpha_{2}^{2}\right)}{\sqrt{1-c_{n}^{2} / \beta_{2}^{2}}}-2\left(2-c_{n}{ }^{2} / \beta_{2}^{2}\right) \sqrt{1-c_{n}^{2} / \alpha_{2}^{2}}\right\}
\end{aligned}
$$

Equation (30) takes the dimensionless form:

$$
\begin{aligned}
& \tan \left(k_{n} H \sqrt{c_{n}^{2} / v_{1}^{2}-1}\right) \\
& \quad=\frac{\rho_{2} \beta_{2}{ }^{4}}{\rho_{1} c_{n}{ }^{4}} \frac{\sqrt{c_{n}^{2} / v_{1}^{2}-1}}{\sqrt{1-c_{n}^{2} / \alpha_{2}^{2}}}\left[4 \sqrt{1-c_{n}^{2} / \alpha_{2}^{2}} \sqrt{1-c_{n}{ }^{2} /{\beta_{2}}^{2}}-\left(2-c_{n}{ }^{2} / \beta_{2}^{2}\right)^{2}\right] .
\end{aligned}
$$

This last equation defines an implicit relationship between the period $T_{n}=2 \pi / c_{n} k_{n}$ and the phase velocity $c_{n}$, with the elastic constants of the system as parameters. It is evident that the wave system given by (28) and (29) will be attenuated for $k_{n}$ complex, the degree of attenuation depending on the magnitude of the imaginary component. The energy loss associated with damped propagation is due to the refraction of waves from the liquid layer into the solid bottom. For the case $\alpha_{2}>\beta_{2}>v_{1}$, which is considered in this paper, undamped propagation will occur for the cases:

$$
\begin{aligned}
\text { I. } & \alpha_{2}>\beta_{2} \geq c \geq v_{1} \\
\text { II. } & \alpha_{2}>\beta_{2}>v_{1} \geq c .
\end{aligned}
$$

Only case I will be considered in detail in this paper.

Numerical calculation of the period from (37) shows it to be a multiplevalued function of phase velocity, each value belonging to a distinct mode of propagation. The summations indicated in (28) and (29) are to be carried over all the modes $n=1$ to $n=\infty$. The amplitude factors $Q\left(k_{n}\right)$ and $W\left(k_{n}\right)$ give the relative excitation of each mode as a function of the period. The factor $e^{-7 n d}$ shows the influence of the depth of the source on the amplitudes of the different modes.

It can be shown ${ }^{17}$ that the period equation (37) expresses the condition of constructive interference between plane waves undergoing multiple reflection in the liquid layer at angles of incidence beyond the critical angle of reflection

\footnotetext{
${ }_{17}$ Frank Press and Maurice Fwing, loc. cit.
} 
$\theta_{c}$, where $\sin \theta_{c}=v_{1} / \beta_{2}$. With this simplified point of view each mode represents a different order of interference and the disturbance at a distant point is obtained by the superposition of waves arriving at the point along the oblique ray paths (defined by the angle of incidence $\theta$ ) for which constructive interference occurs. For the case of plane waves, the wave number $k$ in the previous discussion can be expressed by $k=(2 \pi / l) \sin \theta$, where $l$ is the wave length measured along an oblique ray path. The plane wave theory, using the elementary concept of reflected and refracted waves fails to provide an explanation of how rays from a source in the bottom can enter the liquid and then travel within it at angles of incidence beyond $\theta_{c}$ to provide for the total reflection necessary for normal mode propagation.

Generalization for an arbitrary pulse.-In a dispersive medium in which an arbitrary initial disturbance occurs, the energy associated with each period is known to propagate with the group velocity given by the well-known formula

$$
U=c+(k h) \frac{d c}{d(k h)}
$$

The period equation (37) was used to obtain the phase velocity as a function of $k h$. The group velocity was subsequently determined by numerical differentiation according to (38). The results of these computations for $c \geq v_{1}$ (case $I$ above) are shown in figures 2 and 3 , where $c / v_{1}$ and $U / v_{1}$ are plotted as functions of the dimensionless parameter $\gamma=H / l=H / v_{1} T=$ $k H c / 2 \pi v_{1}$ for the two cases $\rho_{2} / \rho_{1}=2.5, \alpha_{2}=\sqrt{3} \beta_{2}, \beta_{2}=2 v_{1}$ and $\rho_{2} / \rho_{1}=2.5$, $\alpha_{2}=\sqrt{3} \beta_{2}, \beta_{2}=3 v_{1}$, which represent respectively the approximate conditions for a granitic and basaltic bottom. Since the functional dependence of $k h$ on $c / v_{1}$ is not very sensitive to small changes in the value of $\rho_{2} / \rho_{1}$, we have taken a single value of this constant for both cases. For both cases two modes are plotted. In an earlier paper ${ }^{18}$ the first and second modes were considered to represent two distinct branches of the period equation, the second branch having higher modes. This distinction is artifical and unnecessary. The phase and group velocity of the first mode $(n=1)$ approach the velocity $\left(c_{R}\right)$ of Rayleigh waves in the bottom layer as $\gamma \rightarrow 0$, or as the wavelength becomes very long in comparison to the thickness of the first layer. As $c / v_{1}$ and $U / v_{1} \rightarrow 1$, $\gamma$ approaches a limiting value. For $c / y_{1}<1$ (case II above), the propagation reduces to that of a boundary wave (Stoneley wave) along the bottom interface, which exists only where $c / v_{1}$ is very nearly unity. In the second mode $(n=2)$ the phase and group velocity equals the velocity of shear waves in the bottom $\left(c / v_{1}=U / v_{1}=\mathscr{2}\right.$ or $c / v_{1}=U / v_{1}=3$ as the case may be) for a limiting value of $\gamma$ corresponding to a cut-off period aobve which $k$ is complex and damping occurs. For $c$ and $U$ close to $v_{1}$ the periods become infi-

\footnotetext{
${ }^{18}$ Frank Press and Maurice Ewing, loc. cit.
} 
nitely small. Higher modes of propagation $(n=3,4 \ldots)$ exist, each having the same cut-off velocity $c=U=\beta_{2}$, but increasingly shorter cut-off periods. In general the periods of the higher modes corresponding to a given phase or group velocity become progressively shorter. The group velocities of the first and second modes are further characterized by the existence of stationary values 'of group velocity, the importance of which will be discussed in a later paragraph.

Having obtained the solution for the steady state propagation of simple harmonic compressional waves originating in a point source, it remains yet to obtain the solution for an arbitrary initial disturbance. If the time variation of the initial disturbance (19) at the source is $f(t)$ represented by its Fourier transform

$$
f(t)=\frac{1}{2 \pi} \int_{-\infty}^{\infty} e^{i \omega t} g(\omega) d \omega
$$

the component displacements $q_{H}^{\prime}$ and $w_{H}^{\prime}$ for an arbitrary pulse can be written as

$$
\begin{aligned}
q_{H}{ }^{\prime} & =\frac{1}{H^{2}} \sqrt{\frac{2}{\pi r}} \sum_{n} \int_{-\infty}^{\infty} \frac{1}{\sqrt{k_{n}}} Q\left(k_{n}\right) g(\omega) e^{-\eta_{n} d} e^{i\left(\omega t-k_{n} r+\pi / 4\right)} d \omega \\
w_{H}{ }^{\prime} & =\frac{1}{H^{2}} \sqrt{\frac{2}{\pi r}} \sum_{n} \int_{-\infty}^{\infty} \frac{1}{\sqrt{k_{n}}} W\left(k_{n}\right) g(\omega) e^{-\eta_{n} d} e^{i\left(\omega t-k_{n} r-\pi / 4\right)} d \omega
\end{aligned}
$$

where $k_{n}$ is a function of $\omega$ through (30) and $g(\omega)$ is given by

$$
g(\omega)=\int_{-\infty}^{\infty} e^{-i \omega t} f(t) d t
$$

Pekeris ${ }^{19}$ was primarily interested in a source consisting of an exploding charge and he appropriately chose $f(t)=e^{-\sigma t}$ for $t>0$ and $f(t)=0$ for $t<0$. Our primary interest is the impulse developed in an earthquake, and for lack of more detailed information we choose $f(t)$ to vanish for all but infinitesimal values of $t$ in such a manner that $\int_{-\infty}^{\infty} f(t) d t=A$. With this choice, equations (40) and (41) are given by:

$$
\begin{aligned}
q_{H}{ }^{\prime} & =\frac{A}{H^{2}} \sqrt{\frac{2}{\pi r}} \sum_{n} \int_{-\infty}^{\infty} \frac{1}{\sqrt{k_{n}}} Q\left(k_{n}\right) e^{-\eta_{n} d} e^{i\left(\omega t-k_{n} r+\pi / 4\right)} d \omega \\
w_{H}{ }^{\prime} & =\frac{A}{H^{2}} \sqrt{\frac{2}{\pi r}} \sum_{n} \int_{-\infty}^{\infty} \frac{1}{\sqrt{k_{n}}} W\left(k_{n}\right) e^{-\eta_{n} d} e^{i\left(\omega t-k_{n} r-\pi / 4\right)} d \omega
\end{aligned}
$$

${ }^{19}$ C. L. Pekeris, loc. cit. 


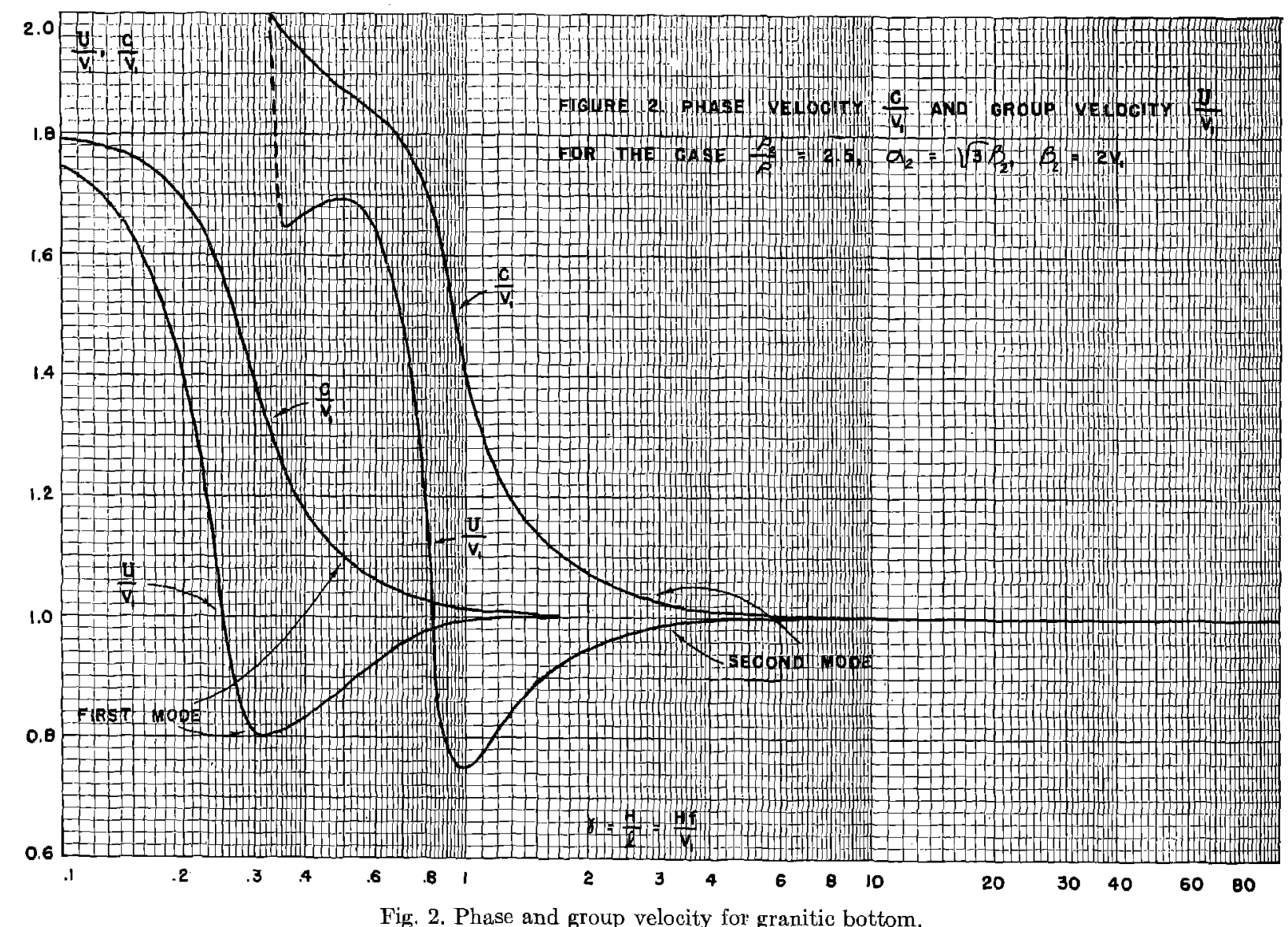




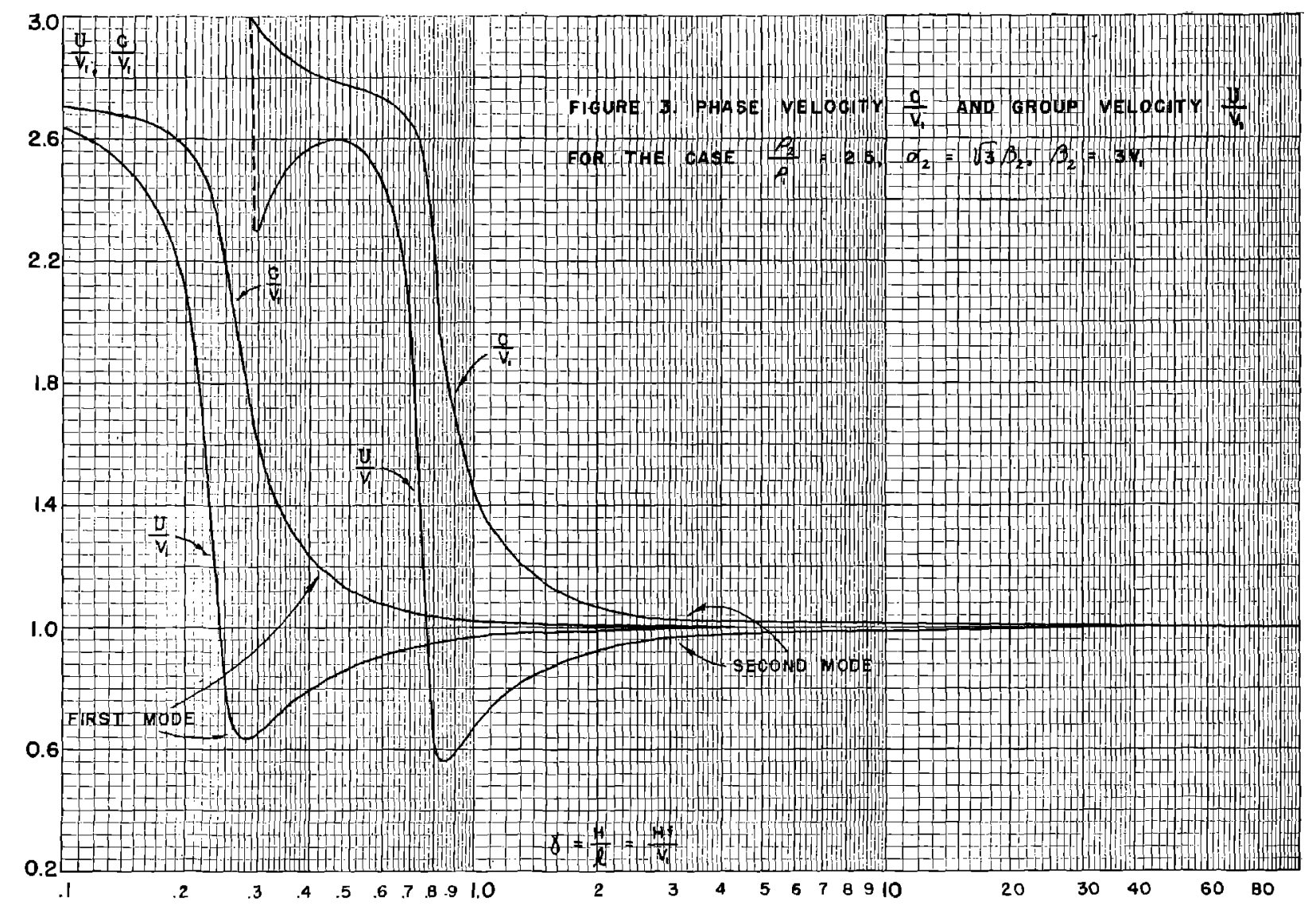

Fig. 3. Phase and group velocity for basaltic bottom. 
To evaluate these integrals, we follow Pekeris in using Kelvin's approximate method of stationary phase. The method assumes that the exponential term oscillates rapidly over the range of integration, whereas the remainder of the integrand is a slowly varying function of $\omega$. The principal contribution to the integral therefore occurs at the points for which $\partial f(\omega) / \partial \omega=0$, where $f(\omega)=$ $\left(\omega t-k_{n} r \pm \pi / 4\right)$. An expansion of $f(\omega)$ about these points of stationary phase is made and formulas for the approximate evaluation of the integral are obtained. Jeffreys, ${ }^{20}$ Lamb, $^{21}$ and Pekeris ${ }^{22}$ derive and discuss the appropriate formulas. In evaluating the integrals (43) and (44) we shall make use of the equations and computations as given by Pekeris. The first of these can be written as follows:

$$
\begin{aligned}
\int_{-\infty}^{\infty} Y(\omega) e^{i(\omega t-k(\omega) r)} d \omega & \simeq \sqrt{\frac{2 \pi}{r\left|\frac{d^{2} k\left(\omega_{0}\right)}{d \omega^{2}}\right|} Y\left(\omega_{0}\right) e^{i\left(w_{0} t-k_{n}\left(\omega_{0}\right) r \pm \pi / 4\right)}} \\
& \simeq \sqrt{\frac{2 \pi}{\left(\frac{H}{2 \pi v_{1}}\right) \frac{r}{U^{2}}\left|\frac{d U}{d \gamma}\right|}} Y\left(\omega_{0}\right) e^{i\left(\omega_{0} t-k\left(\omega_{0}\right) r \pm \pi / 4\right)}
\end{aligned}
$$

where the upper or lower sign is to be taken in the exponential according as $d U / d \gamma$ is positive or negative and $k, U, d U / d \gamma, \omega_{0}$ are evaluated from the group and phase velocity curve for the values of $r$ and $t$ which satisfy $r / t=U$. In deriving the second equation of (45) use was made of the relations $\gamma=\frac{\omega H}{2 \pi v_{1}}$, $U=\frac{d \omega}{d k}, \omega=c k$. The formula given above is valid only when the following condition is met:

$$
\frac{1}{96 \pi^{2}} \frac{H}{r}\left[-\frac{5\left(\frac{d Z}{d \gamma}\right)^{2}}{Z^{3}}+\frac{3 \frac{d^{2} Z}{d \gamma^{2}}}{Z^{2}}\right] \ll<1
$$

where

$$
Z=\frac{v_{1}^{2}}{H} \frac{d^{2} k}{d \omega^{2}}=-\frac{v_{1}}{2 \pi U^{2}} \frac{d U}{d \gamma}
$$

This condition is violated for small $r$ and for points near a stationary value of group velocity. In the latter case we use an approximate formula devised by Airy:

$$
\int_{-\infty}^{\infty} Y(\omega) e^{i(\omega t-k(\omega) r)} d \omega \simeq \frac{2 \pi}{3^{2 / 3}} \frac{E(v) Y\left(\omega_{0}\right) e^{i\left(\omega_{0} t-k\left(\omega_{0}\right) r\right)}}{\left.r^{l / 3}\left[\frac{H^{2}}{2 \pi v_{1}^{3}}\left|\frac{d Z}{d \gamma}\right|\right]\right]^{1 / 3}}
$$

\footnotetext{
${ }^{20}$ Harold Jeffreys, loc. cit. 398.

Horace Lamb, Hydrodynamics (6th ed.; Cambridge University Pंress, 1932), pp. 395-

${ }^{22}$ C. I. Pekeris, loc. cit.
} 
where

and

$$
\begin{aligned}
& E(v)=v^{1 / 3}\left[J_{-1 / 3}(v)+J_{1 / 3}(v)\right]\left\{\begin{array}{l}
t<\frac{r}{U_{\min }} \\
t>\frac{r}{U_{\max }}
\end{array}\right. \\
& E(v)=v^{1 / 3}\left[I_{-1 / 3}(v)-I_{1 / 3}(v)\right]\left\{\begin{array}{l}
t>\frac{r}{U_{\min }} \\
t<\frac{r}{U_{\max }}
\end{array}\right.
\end{aligned}
$$

$$
\begin{array}{ll}
v= & \frac{4 \sqrt{\pi}}{3 \sqrt{\left|\frac{d Z}{d \gamma}\right|}}\left(\frac{r}{H}\right)\left|\tau-\tau_{m}\right|^{3 / 2} \\
\tau=\frac{t v_{1}}{r}-1, & \tau_{m}=\frac{v_{1}}{U_{0}}-1
\end{array}
$$

Only the real part of the integral in (48) has been considered. In these equations the subscript 0 indicates that the quantity to which it is attached is to be evaluated at a stationary value (min. or max.) of group velocity. The use of (48) is justified only when

where

$$
-\frac{1}{(2 \pi)^{2 / 3}}\left(\frac{H}{r}\right)^{1 / 3} \frac{\left(\frac{d^{2} Z}{d \gamma^{2}}\right) G(v)}{\left|\frac{d Z}{d \gamma}\right|^{4 / 3} E(v)}<<1
$$

$$
\begin{array}{lr}
G(v)=\frac{3^{1 / 3}}{4}\left\{-\frac{2}{3} v^{2 / 3}\left[J_{-2 / 3}(v)-J_{2 / 3}(v)\right]+\frac{1}{2} v^{5 / 3}\left[J_{-1 / 3}(v)+J_{1 / 3}(v)\right]\right\} & t<\frac{r}{U_{\text {min }}} \\
& t>\frac{r}{U_{\max }} \\
G(v)=\frac{3^{1 / 3}}{4}\left\{-\frac{2}{3} v^{2 / 3}\left[I_{-2 / 3}(v)-I_{2 / 3}(v)\right]+\frac{1}{2} v^{5 / 3}\left[I_{-1 / 3}(v)-I_{1 / 3}(v)\right]\right\} & t>U_{\min } \\
& t<U_{\max }
\end{array}
$$

We now apply these results to equations (43) and (44). In order to compute the displacements $q_{H}{ }^{\prime}$ and $w_{H}{ }^{\prime}$, one first finds the contribution of the $n$th mode at a given time $t$ and range $r$ by determining $\omega, c / U_{1}, k h,\left(d U / \nu_{1}\right) / d \gamma$ from the 
phase and group velocity curves appropriate to the medium such that $r / t=U$. The displacements $q_{H}^{\prime}$ and $w_{H}^{\prime}$ are then obtained by summing over all modes:

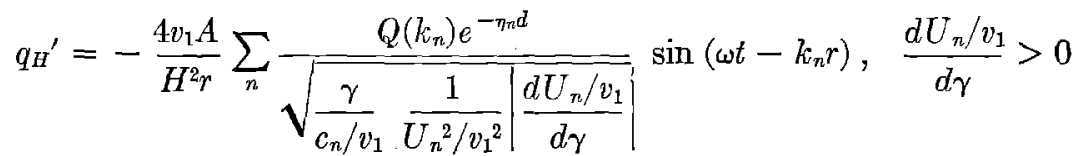

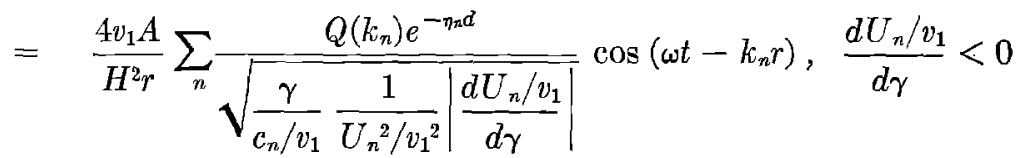

$$
\begin{aligned}
& w_{H}^{\prime}=\frac{4 v_{1} A}{H^{2} r} \sum_{n} \frac{W\left(k_{n}\right) e^{-\eta_{n} d}}{\sqrt{\frac{\gamma}{c_{n} / v_{1}}} \frac{1}{U_{n}^{2} / v_{1}^{2}}\left|\frac{d U_{n} / v_{1}}{d \gamma}\right|} \cos \left(\omega t-k_{n} r\right), \quad \frac{d U_{n} / v_{1}}{d \gamma}>0 \\
& =\frac{4 v_{1} A}{H^{2} r} \sum_{n} \frac{W\left(k_{n}\right) e^{-\eta_{n} d}}{\sqrt{\frac{\gamma}{c_{n} / v_{1}} \frac{1}{U_{n}^{2} / v_{1}^{2}}\left|\frac{d U_{n} / v_{1}}{d \gamma}\right|}} \sin \left(\omega t-k_{n} r\right), \quad \frac{d U_{n} / v_{1}}{d \gamma}<0,
\end{aligned}
$$

provided $r$ is large and $t$ is sufficiently removed from the value $t_{0}=r / U_{0}$, where $U_{0}$ denotes a stationary value of group velocity. We have added to the expressions (43) and (44) their complex conjugates in deriving (56)-(59), since $Q$ and $W$ are even functions of $\omega, f(\omega)$ is an odd function of $\omega$, and there are two stationary points $\pm \omega$ where $\partial f(\omega) / \partial \omega=0$.

Near the time $t_{0}$ corresponding to a stationary value of group velocity (Airy phase) one first determines $\gamma, \omega, k, c, Z, d Z / d \gamma$ at this point from the phase and group velocity curves appropriate to the medium. As before, $q_{H}{ }^{\prime}$ and $w_{H}{ }^{\prime}$ are then computed by summing over all modes:

$$
\begin{aligned}
q_{H^{\prime}} & =\frac{4 v_{1} A}{3^{2 / 3} H^{2} r^{5 / 6}} \sum_{n} \frac{Q\left(k_{n}\right) e^{-\eta_{n} d} E(v)}{\sqrt{k / 2 \pi}\left[\frac{H^{2}}{2 \pi}\left|\frac{d Z}{d \gamma}\right|\right]^{1 / 3}} \cos \left(\omega t-k_{n} r+\pi / 4\right) \\
w_{H}^{\prime} & =\frac{4 v_{1} A}{3^{2 / 3} H^{2} r^{5 / 6}} \sum_{n} \frac{W\left(k_{n}\right) e^{-\eta_{n} d} E(v)}{\sqrt{k / 2 \pi}\left[\frac{H^{2}}{2 \pi}\left|\frac{d Z}{d \gamma}\right|\right]^{1 / 3}} \cos \left(\omega t-k_{n} r-\pi / 4\right),
\end{aligned}
$$

provided $r$ is large and $t$ sufficiently close to $t_{0}$ for condition (53) to hold. The factors $E(v)$ from which the envelop of the Airy phase can be determined and $\mathrm{G}(v) / E(v)$ from which the range of validity of (60) and (61) can be found were plotted by Pekeris and are reproduced here as figure 4 .

Equations (56) through (61) have been used to compute the amplitudes of the component displacements at the bottom interface for an impulsive point 


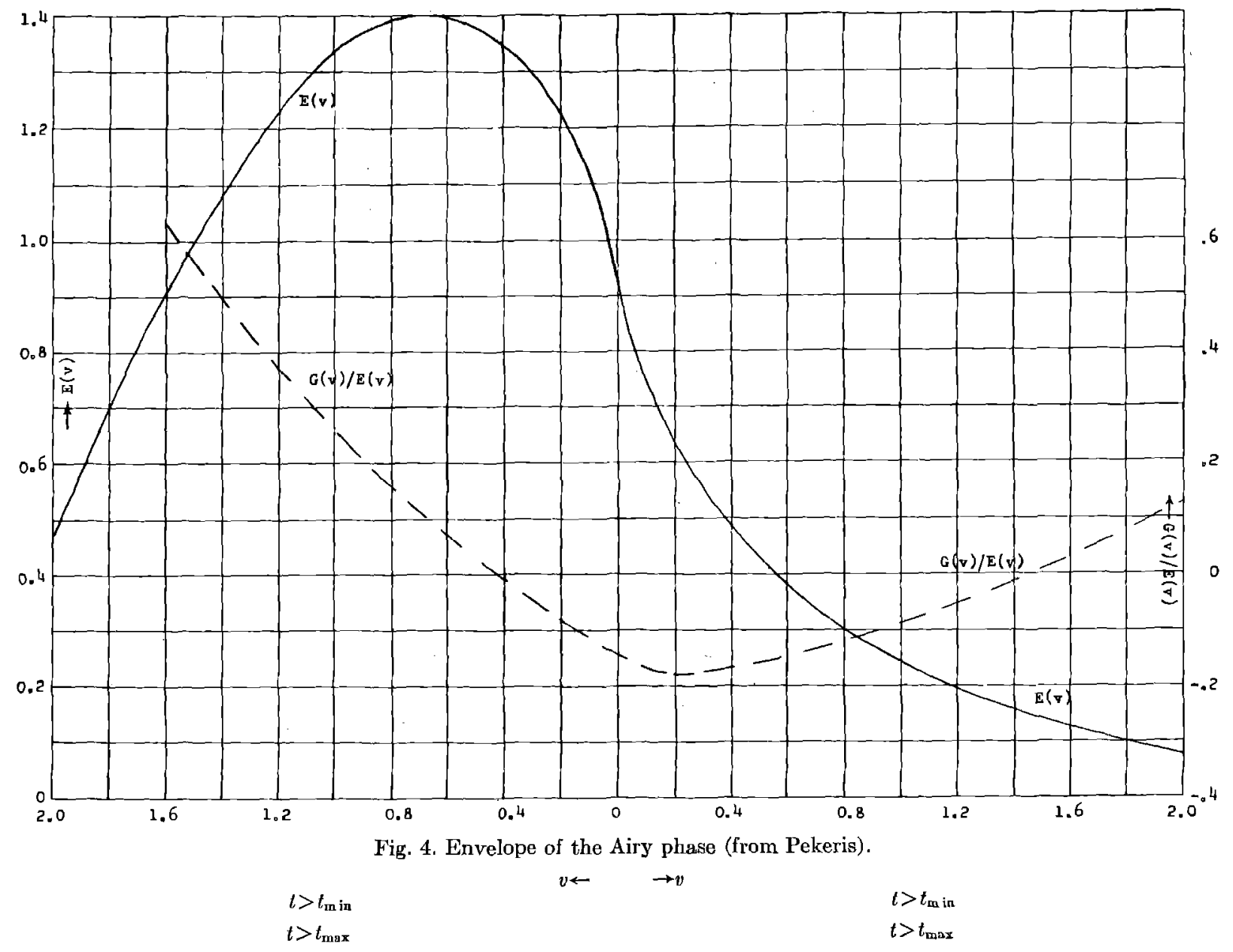




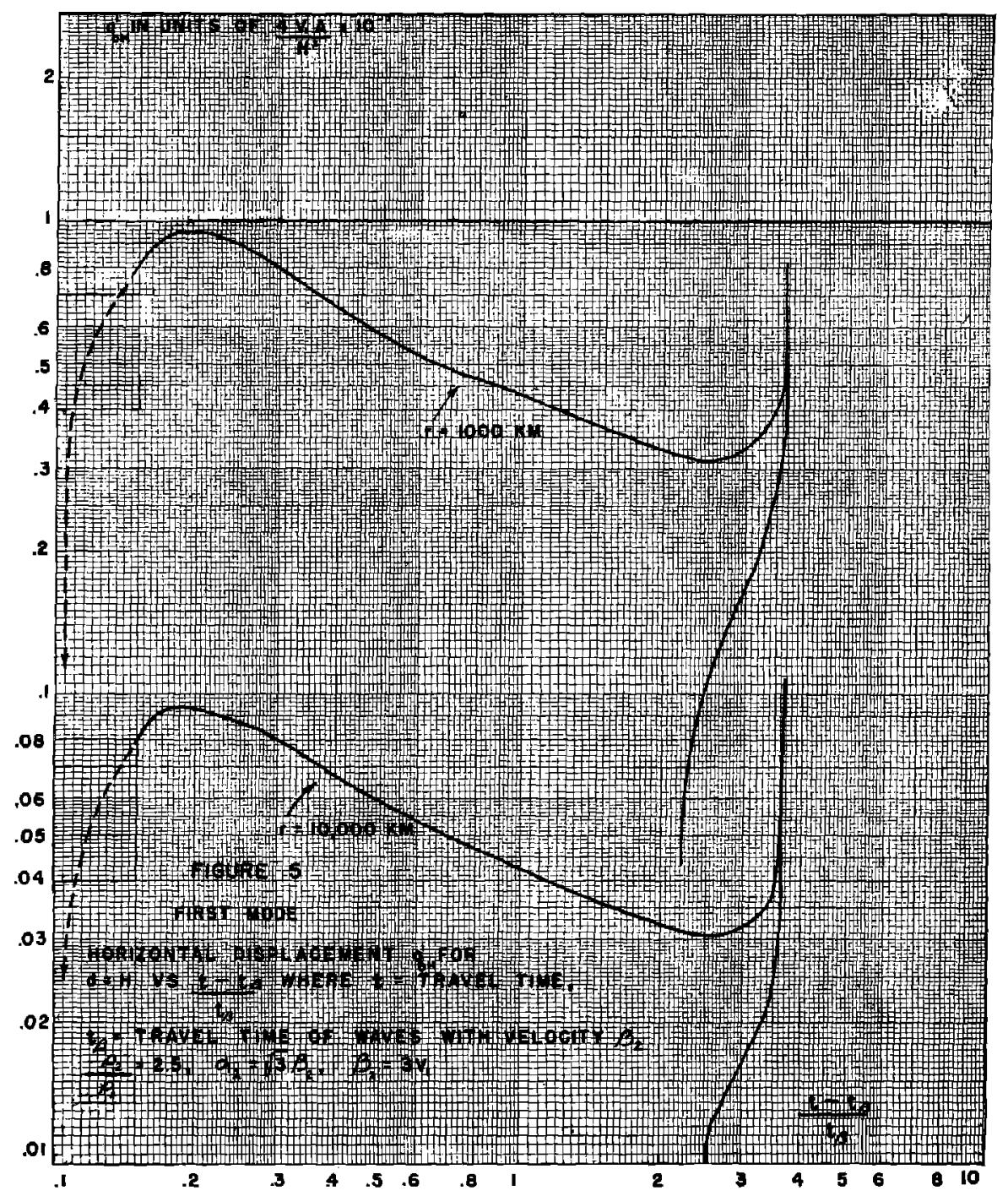

Fig. 5. Horizontal displacement, first mode.

source of compressional waves located at a depth beneath the interface equal to the depth of the water $(d=H)$ and at distances of $1,000 \mathrm{~km}$. and 10,000 $\mathrm{km}$. respectively. The results for the first and second modes are presented in figures 5 to 8 for the case

$\frac{\rho_{2}}{\rho_{1}}=2.5, \quad \alpha_{2}=\sqrt{3} \beta_{2}, \quad \beta_{2}=3 v_{1}, \quad H=5 \mathrm{~km} ., \quad r=1000 \mathrm{~km} ., \quad 10,000 \mathrm{~km}$. $\rho_{1}$ 


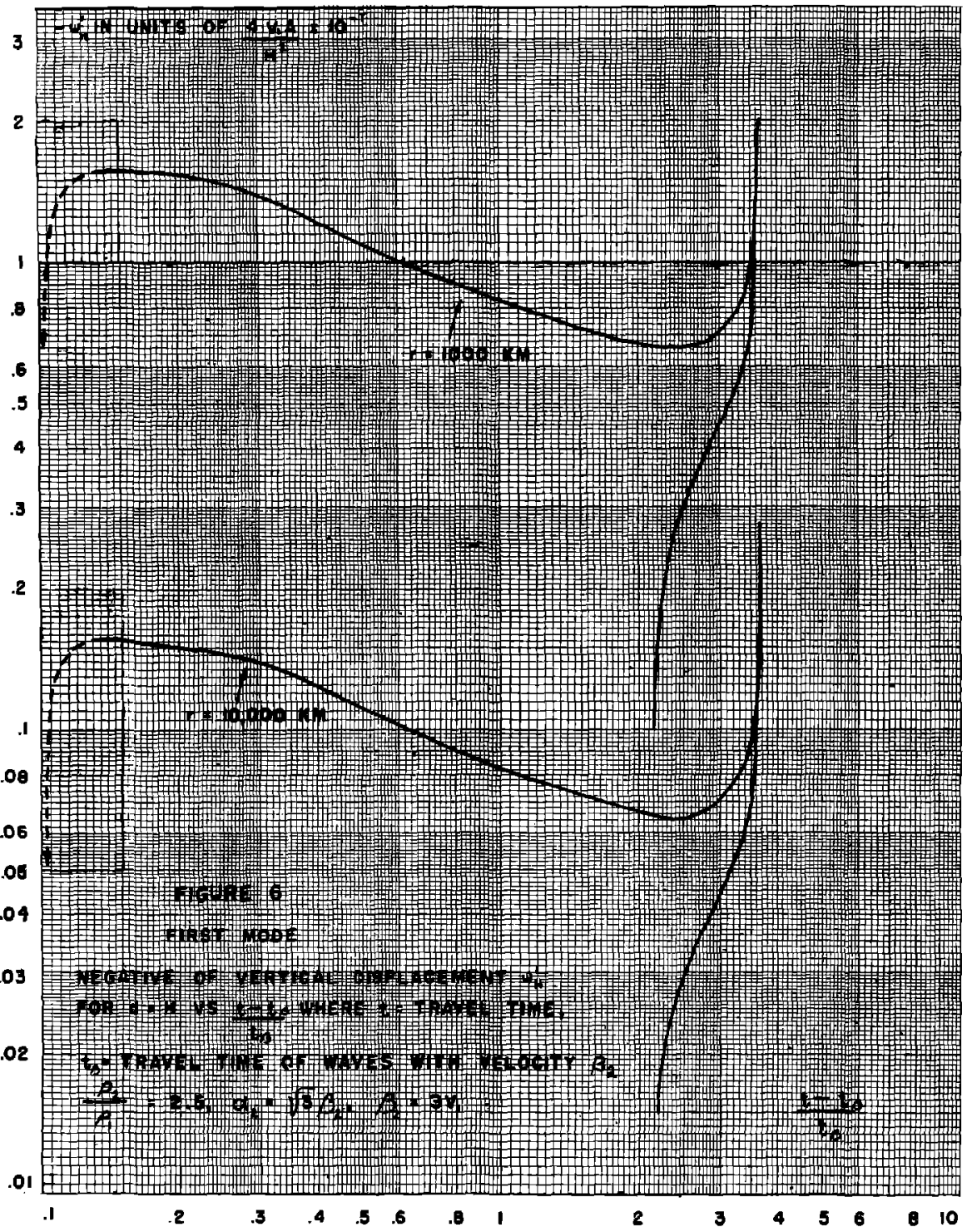

Fig. 6. Vertical displacement, first mode. 


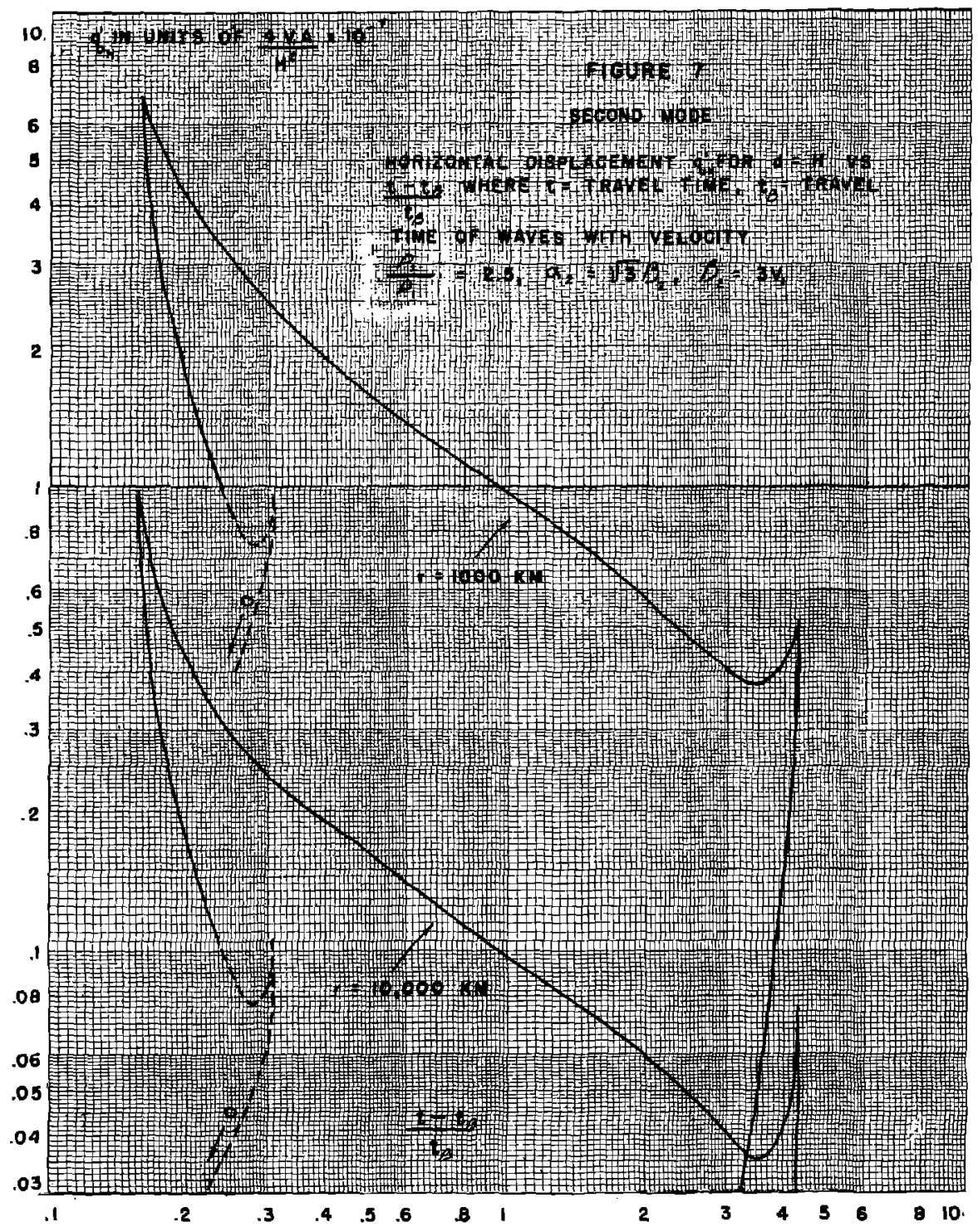

Fig. 7. Horizontal displacement, second mode. 


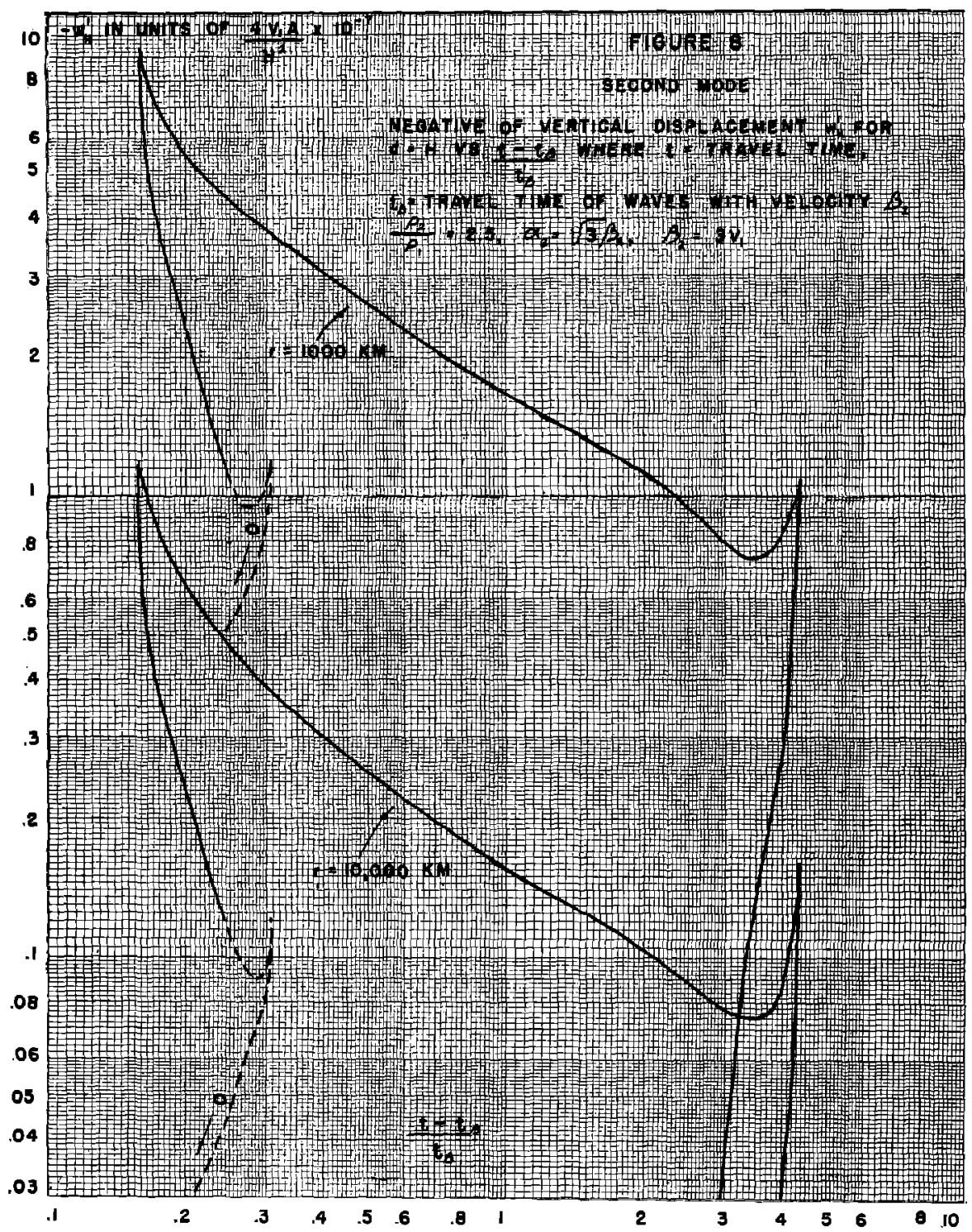

Fig. 8. Vertical displacement, second mode. 


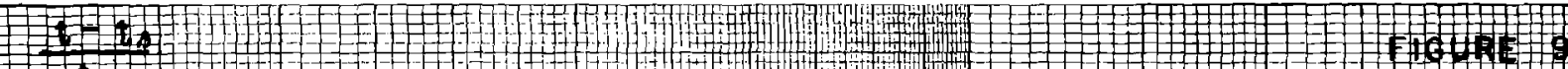

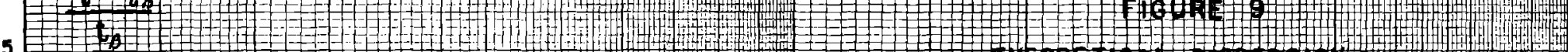

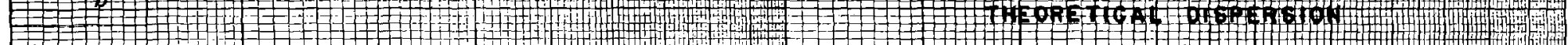
- 7 it L

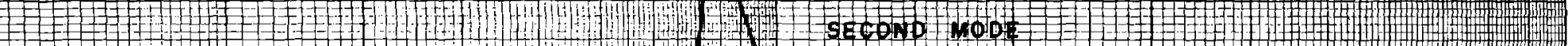
(1)

3

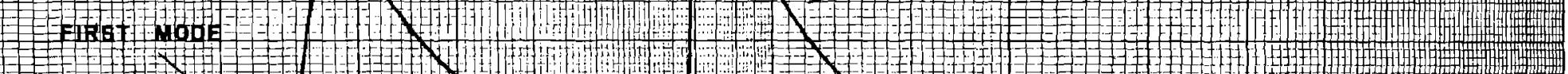

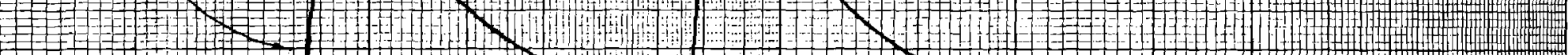
-

2 -

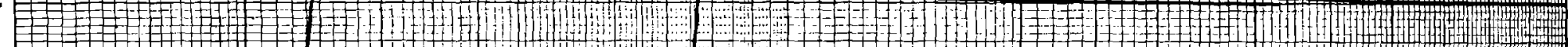

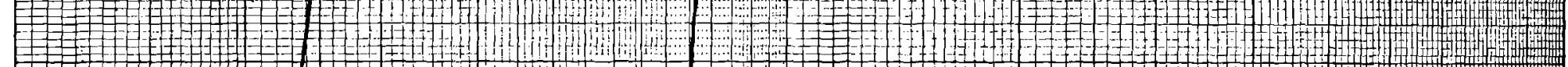

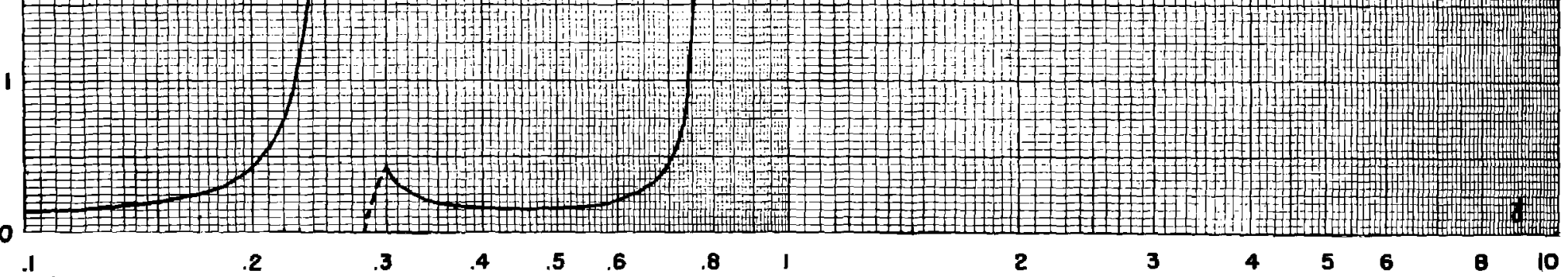
Fig. 9. Theoretical dispersion, first and second modes. 


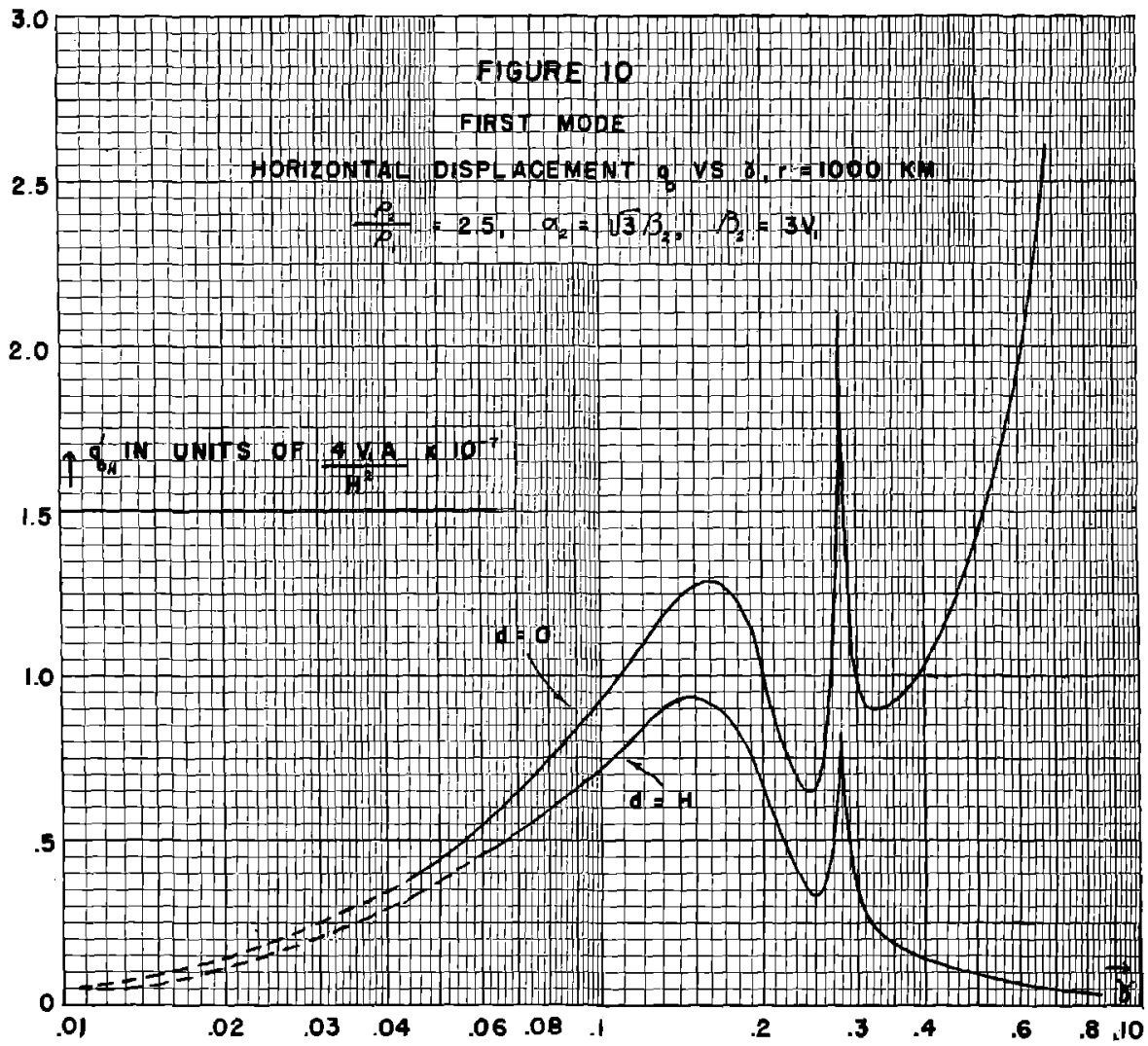

Fig. 10. Horizontal displacement, first mode.

In these figures the amplitudes are plotted as functions of the dimensionless parameter $\frac{t-t_{\beta}}{t_{\beta}}$, where $t$ denotes the time after the initial impulse and $t_{\beta}$ is the travel time for shear waves in the bottom. The travel time of waves of any given period in the first two modes can be computed from figure 9 .

In order to show the effect of the depth of the source on the displacements $q_{H}^{\prime}$ and $w_{H}^{\prime}$, the amplitudes were computed for a source located in the bottom layer close to the interface $(d=0)$. The resultant amplitudes in the first two modes for the two cases $d=0$ and $d=H$ are plotted against $\gamma=H / l$ in figures 10 to 13 .

Discussion of the normal-mode solutions.-In an earlier section the formal steady-state solutions (26) and (27) were expressed as the sum of the residues of the integrands and two integrals along branch lines corresponding to the branch points $c=\alpha_{2}$ and $c=\beta_{2}$. The residues lead to the normal-mode solu- 


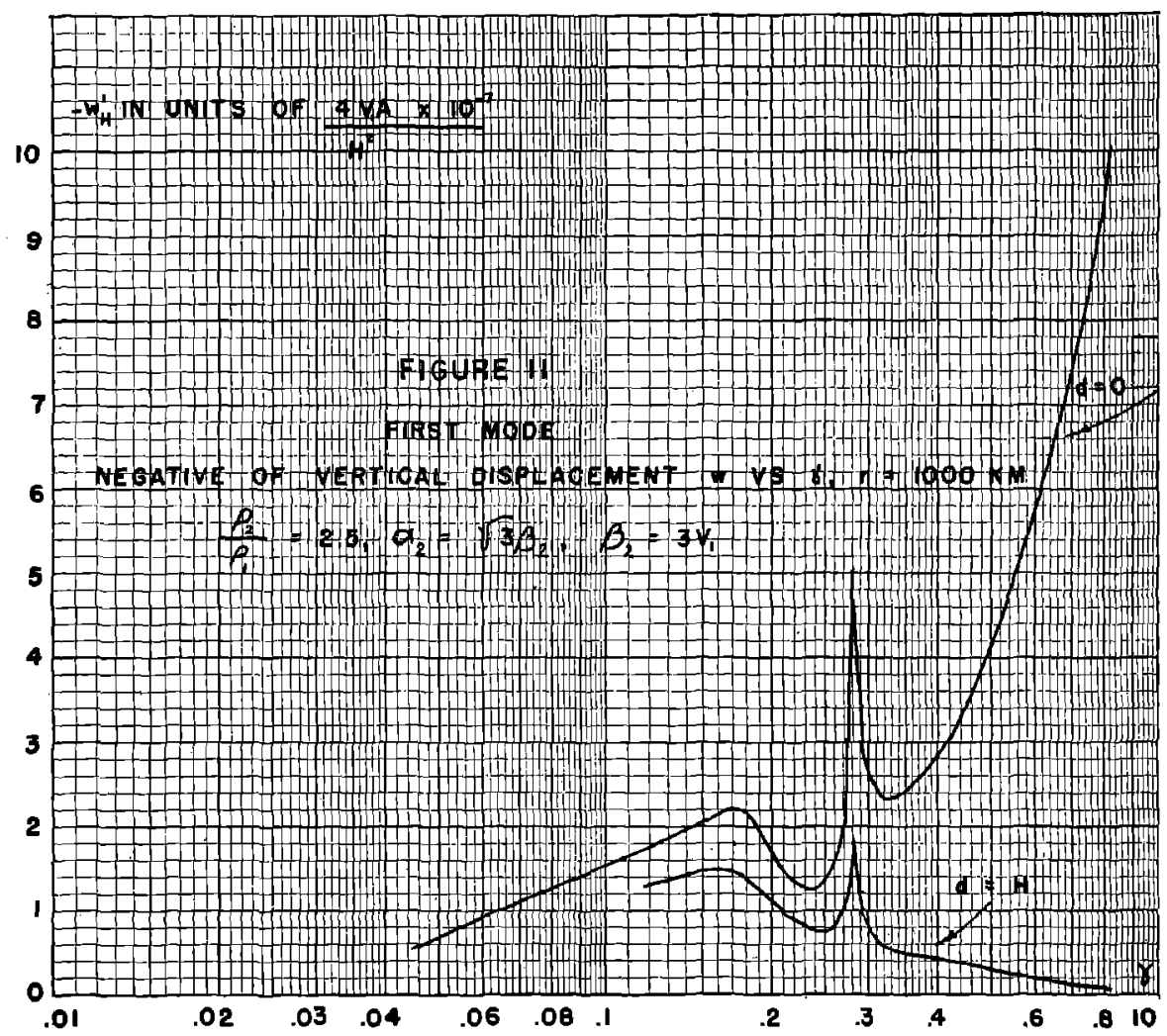

Fig. 11. Vertical displacement, first mode.

tions which predominate at large distances because they diminish only as $1 / \sqrt{r}$. Now the normal-mode solutions vanish at $c=U=\beta_{2}$ and do not exist for $c>\beta_{2}$. In this region the branch-line integrals contribute waves traveling with the speed of compressional and shear waves in the bottom, diminishing rapidly in amplitude with distance as $1 / r^{2}$.

At a time $t>r / \beta_{2}$ after the initial impulse (i.e., immediately after the arrival of shear waves) the normal mode solutions predominate providing $r$ is large. Thus the ground motion in the first two modes due to a distant impulsive point source of compressional waves having a moderately broad spectrum and located within the bottom can be obtained with the aid of figures 3 and 5-9.

In the first mode the group velocity approaches the velocity of Rayleigh waves $\left(c_{\mathrm{R}}=.9194 \beta_{2}\right)$ as $\gamma \rightarrow 0$. The first arrival consists of long-period Rayleigh waves whose amplitudes and period vary with time according to the graphs of figures 5,6 , and 9 . At the time $t=r / v_{1}$ a short-period wave traveling 


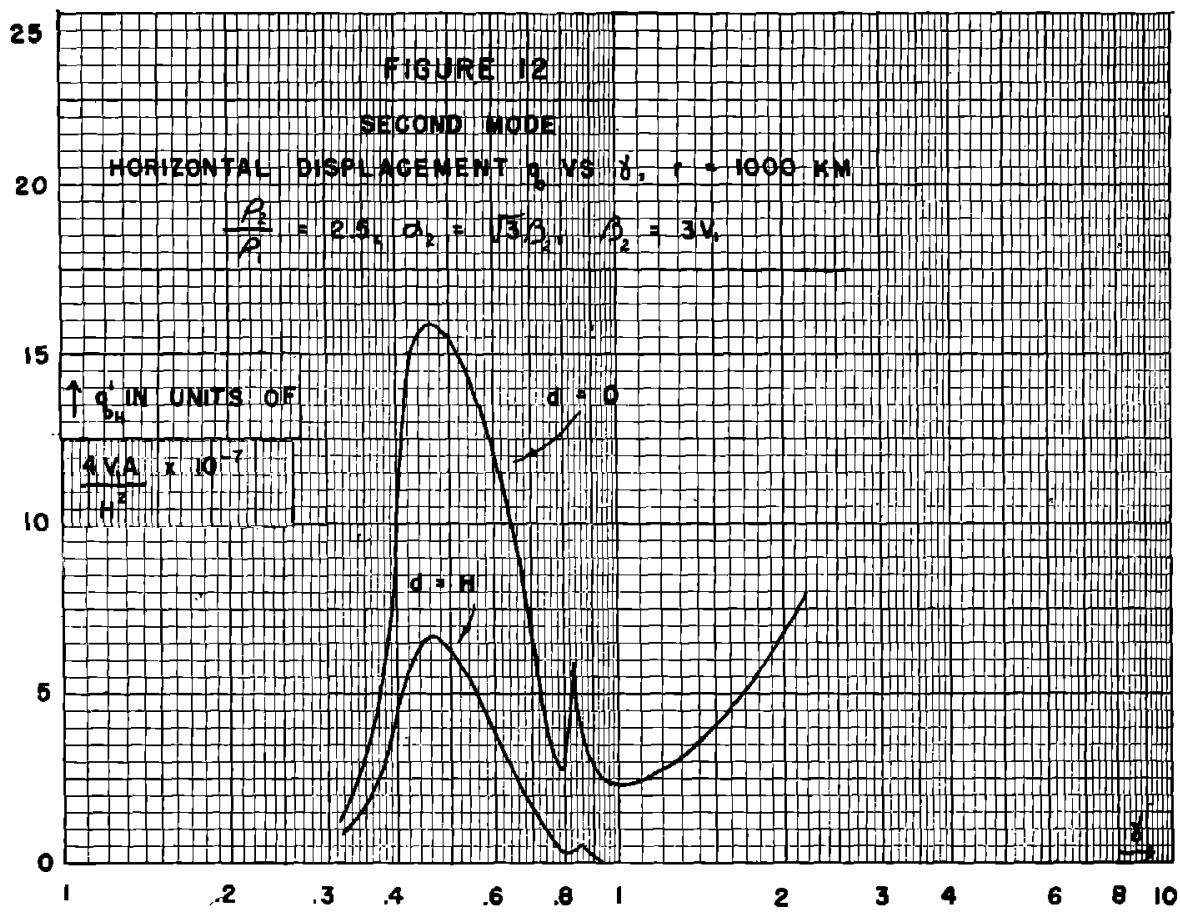

Fig. 12. Horizontal displacement, second mode.

with the speed of sound in water arrives. ${ }^{23}$ According to figures 10 and 11 , amplitudes associated with this wave are particularly small unless the source is located close to the interface. This follows from the factor $e^{-\eta_{n} d}$ in equations (56)-(61), which gives the dependence of the normal-mode amplitudes on the depth of the source beneath the interface. Since

$$
\eta_{n} d=k_{n} H\left(\frac{d}{H}\right) \sqrt{1-c_{n}^{2} / \alpha_{2}^{2}}=\frac{2 \pi}{c / v_{1}} \gamma_{n}\left(\frac{d}{H}\right) \sqrt{1-c_{n}^{2} / \alpha_{2}^{2}}
$$

the amplitudes of the shorter-period waves ( $\gamma_{n}$ large) are cut down if the source has any depth whatever. For source depths greater than several times the depth of water the amplitudes of all but the longest-period waves are reduced to insignificantly small values.

For $t>r / v_{1}$ the short-period and long-period branches of the group velocity curve approach each other and finally merge at a minimum value. The Airy

${ }^{23}$ The group velocity curves show an upper frequency limit for these waves. For higher frequencies the solutions reduce to boundary waves propagated along the interface in a manner analagous to Stoneley waves. 


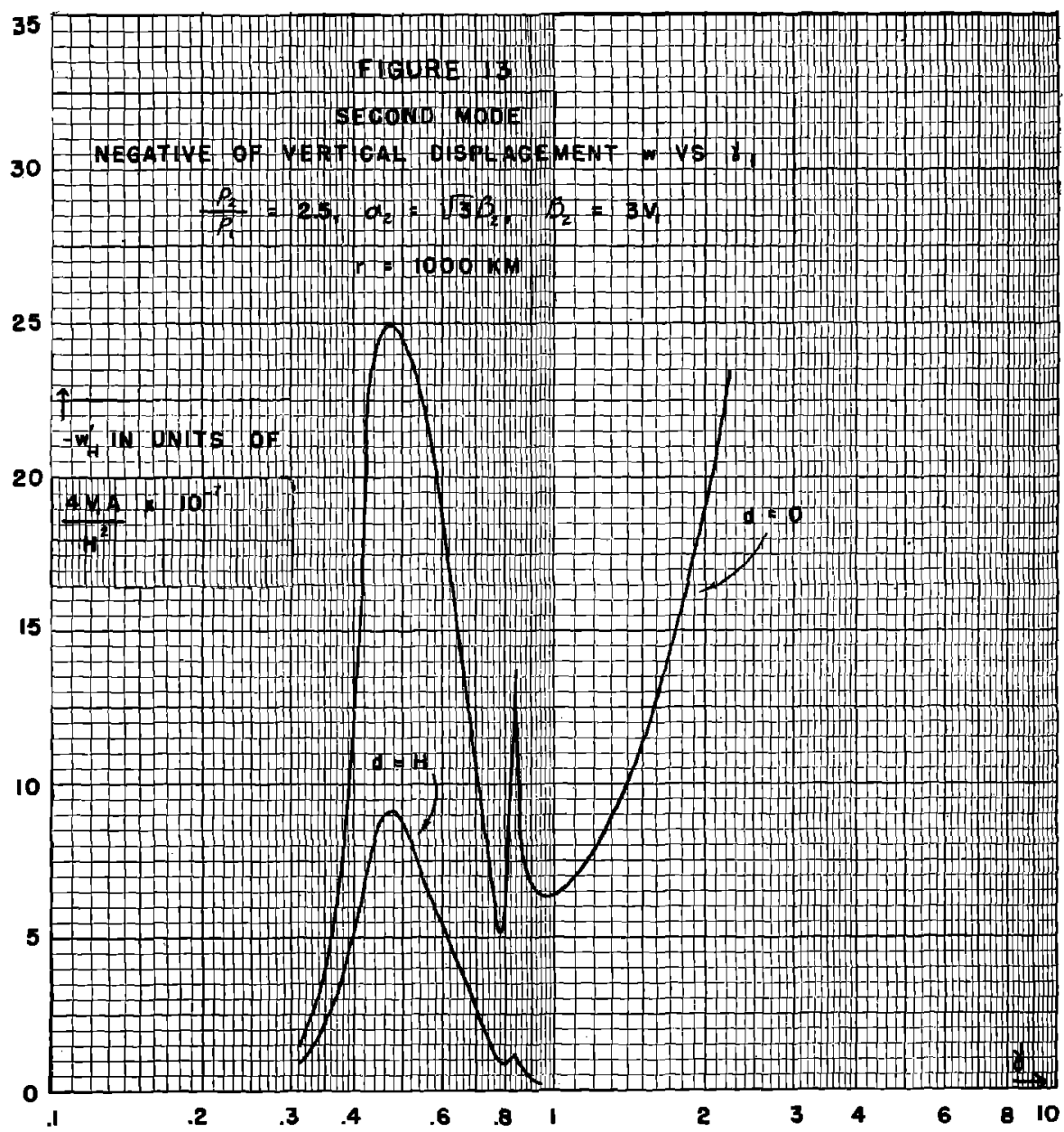

Fig. 13. Vertical displacement, second mode.

phase associated with this stationary value of group velocity consists of waves of large amplitude having a prescribed period and velocity. Since waves propagated at a stationary value of group velocity decrease with distance as $1 / r^{5 / 6}$ (equations 60 and 61) and waves propagated at other values of group velocity diminish as $1 / r$, the relative amplitude of the Airy phase increases with distance. This effect is shown in figures 5 and 6 , where the amplitudes have been computed for the two ranges $r=1000 \mathrm{~km}$. and $r=10,000 \mathrm{~km}$.

The ground motion in the second mode (figs. $7,8,12,13$ ) begins with waves arriving with a cut-off period at a speed $U=\beta_{2}$. The amplitudes are zero at the onset and thereafter increase. At a time corresponding to propagation 
at the maximum value of group velocity ${ }^{24}$ large-amplitude waves arrive with an abrupt onset. Thereafter these waves separate into several simultaneous arrivals of different periods and amplitudes. At the time $t=r / v_{1}$ a short-period wave $(\gamma \rightarrow \infty)$ arrives traveling with the speed of sound in water. As was the case in the first mode, the amplitude of this phase is negligibly small unless the source is located at or very near to the interface. For $t>r / v_{1}$ the two arrivals corresponding to two branches of the group velocity curve approach each other and merge at a minimum value of group velocity, producing an additional Airy phase in the second mode. Since the second mode Airy phase occurs at a shorter period, its amplitude is smaller than that of the first mode if the source depth is of the order of the thickness of the liquid layer or greater.

Table 1 gives the period $T$ and group velocity $U / v_{1}$ associated with each of the stationary values of group velocity of the first two modes for the two cases (a) $\rho_{2} / \rho_{1}=2.5, \alpha_{2}=\sqrt{3} \dot{\beta}_{2}, \beta_{2}=2 v_{1}, v_{1}=1520$ meters $/$ sec., $H=5000$ meters, and (b) $\rho_{2} / \rho_{1}=2.5, \alpha_{2}=\sqrt{3} \beta_{2}, \beta_{2}=3 v_{1}, v_{1}=1520$ meters $/$ sec.,

TABLE 1

Pertods and Group Velocities of Ainy Phases

\begin{tabular}{c|c|c|c|c}
\hline & & $\gamma$ & $T$ & $U / v_{1}$ \\
\hline \multirow{4}{*}{ Case a } & First mode & .33 & $10 \mathrm{sec}$ & .80 \\
\cline { 2 - 5 } & Second mode & .49 & 6.7 & 1.7 \\
& .98 & 3.4 & .75 \\
\hline & & .28 & 12 & .64 \\
\hline & First mode & .28 & .6 \\
\cline { 2 - 4 } & Second mode & .46 & 7.2 & 2.6 \\
& & .82 & 4.0 & .56 \\
\hline
\end{tabular}

$H=5000$ meters. The minimum value of group velocity in the second mode indicated by the dashed lines in figures 2 and 3 is not included in table 1 since its exact position has not been calculated. We infer that amplitudes associated with this point are not significantly large because of its proximity to the cut-off period and its extremely sharp curvature.

The ratio of vertical to horizontal displacement for the first two modes is plotted as a function of $\gamma$ in figure 14. It is seen that for $\gamma \rightarrow 0$ the ratio $w / q$ approaches the correct value for Rayleigh waves propagated along the surface of a semi-infinite elastic solid for which $\lambda=\mu$.

From equations (2), (3), and (23) it can be seen that the factor $\sin \left(\xi_{n} z\right)$ gives the vertical variation of pressure and horizontal displacement and

\footnotetext{
${ }^{24}$ Pekeris attaches the name Airy phase in reverse to maximum values of group velocity.
} 


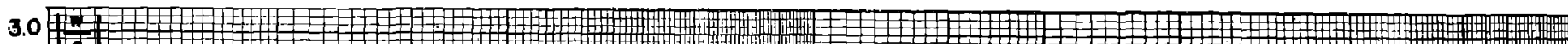
(1) \begin{tabular}{l}
1 \\
\hline
\end{tabular} \begin{tabular}{lll}
\hline \\
\hline
\end{tabular}

2.5 I (1)

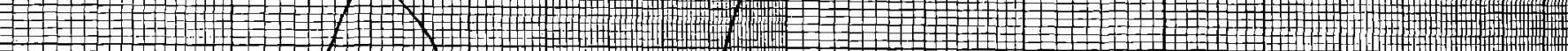
-

2.0 2 Z -

5 (3) F-1

1.0

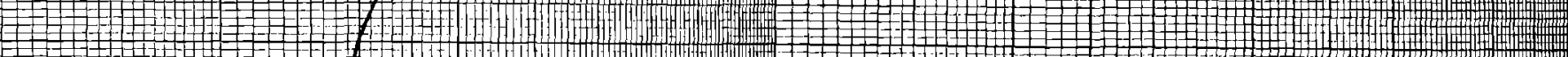

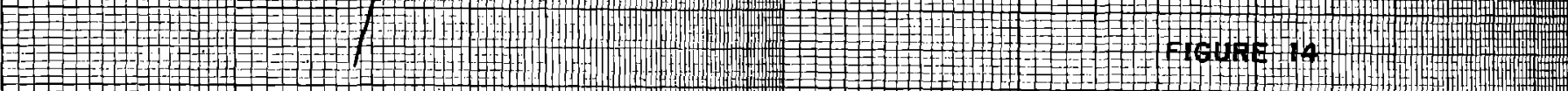
= 5 - 1 红 彭

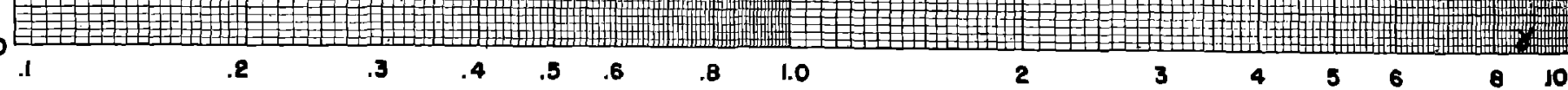
Fig. 14. Ratio of vertical to horizontal displacement, first and second modes. 
$\cos \left(\xi_{n} z\right)$ gives the vertical variation of vertical displacement in the liquid layer. Nodes of pressure correspond to antinodes of vertical displacement, and vice versa. In figure 15 the vertical pressure variation in the liquid layer is presented as a function of $\gamma$ for the first two modes.

The ground motion at a point is obtained by the superposition of the contributions of all modes. Our discussion thus far has been limited to the first two modes. The periods of the higher modes corresponding to a given group velocity become progressively shorter. For source depths of the order of a fraction of the depth of water or more, the higher modes will contribute little to the ground motion because of the factor $e^{-\eta_{n} d}$. In addition, the contribution of the short period higher modes will be cut down because of their greater susceptibility to scattering by an irregular ocean bottom. The poor shortperiod response characteristics of intermediate and long-period seismographs will further discriminate against the higher modes.

The theory and computations described represent an earthquake by an impulsive point source of compressional waves. Actually an earthquake is neither of these. A natural source is usually extended and rich in distortional waves.

Despite the highly idealized assumptions concerning the nature of an earthquake source, it is believed that the normal mode theory given above is sufficiently adequate to explain the salient features on the seismograms of shallowfocus submarine earthquakes.

Thus far, only the Airy phase of the first mode and the short-period waterwave phase (T phase) have been observed. In the next section the data on the Airy phase are presented. The T phase has been described in an earlier report. ${ }^{25}$

\section{The Airy Phase Observed at Bermuda and Huancayo}

Bermuda.-The Bermuda seismological station is situated at $32^{\circ} 23^{\prime} \mathrm{N}$, $64^{\circ} 41^{\prime} \mathrm{W}$. It is equipped with a Milne-Shaw, two components, oriented NE-SW and NW-SE. The free period is given as 12 sec. and the magnification as 250 by the U.S.C.G.S. seismological bulletin.

A series of records was examined in the hope of detecting late arrivals of 9-12 sec. period. Such arrivals were detected on all readable seismograms of the Dominican Republic shocks, but usually not for shocks occurring elsewhere in the Atlantic. Thus a series of Mid-Atlantic Ridge tremors were studied, including the large shocks of April $11,1946\left(1^{\circ} \mathrm{S}, 141 / 2{ }^{\circ} \mathrm{W}\right.$, Pasadena magnitude 7.2), and November 25, $1941\left(371 / 2^{\circ} \mathrm{N}, 1812^{\circ} \mathrm{W}\right.$, magn. 81/4), and with one exception all gave negative results. We will dwell upon this point later.

Table 2 gives the results obtained for a series of Dominican Republic shocks. $H$ is the origin time, $M_{\mathrm{A}}$ gives the arrival time of the maximum amplitude

${ }^{25}$ Ivan Tolstoy and Maurice Ewing, “The T phase of Shallow-focus Earthquakes," Bull. Seism. Soc. Am., 40:25-51 (1950). 

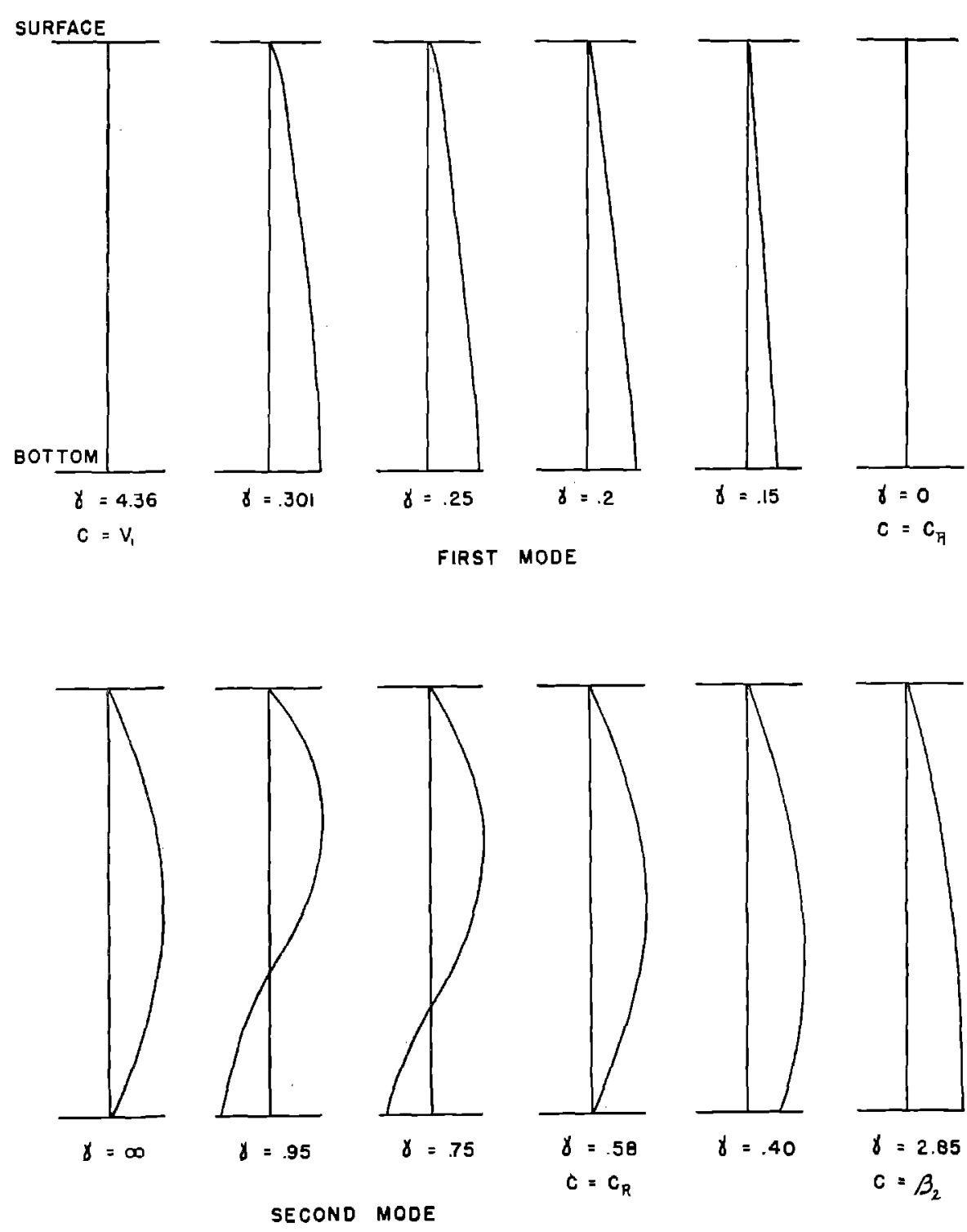

Fig. 15. Vertical pressure distribution as a function of $\gamma$. 
wave of the group interpreted as being the Airy phase. It will be noted that in some cases these times appear to differ somewhat for the two components. The column marked "t.t." gives the total travel time of the Airy phase in minutes and seconds. $A_{\mathrm{A}}$ gives the maximum double-trace amplitude of the Airy phase; $A_{\mathrm{P}}$ and $A_{\mathrm{s}}$, those of the $\mathrm{P}$ and $\mathrm{S}$ groups (in $\mathrm{cm}$.). $T$ gives the period of the group on the seismogram in seconds. This, as well as the arrival times, are only approximate, since the paper velocity of these seismograms is only 8 $\mathrm{mm} / \mathrm{min}$. However, they are sufficient for the present purposes.

This list includes only shocks of magnitude 7 or less. The large shocks, such as those of August 8, 1946 (magn. 7.6), and August 4, 1946 (magn. 8), July 29, 1943 (magn. 7.3), and others, are not included. The reason for this is the impossibility of distinguishing the phase we are studying, owing to the very large trace amplitudes and consequent confusion and fading of the traces.

Arrival times and velocities.-Let us see now what these arrival times correspond to. The times given in table 2 were read for the maximum amplitude of the group of waves interpreted as Airy phases. Such a procedure is justified by the theory of wave propagation in dispersive media: for a stationary value of group velocity there is a gradual buildup of the amplitudes until the arrival of the corresponding wave length, at which point the amplitude is maximum.

For purposes of comparison of the arrival times of this phase with those of the classical earthquake phases it would be necessary to pick the time at the beginning of the group. For this one should subtract roughly $2 \mathrm{~min}$. from the preceding arrival times.

The first remark that we shall make is that, even with the latter provision, the travel times are much too large to be any of the known tabulated earthquake phases ${ }^{26}$ such as multiply reflected body waves or Love and Rayleigh waves. They are too large for $\mathrm{ScS}$ even if this could be observed at such a short distance from the epicenter.

Also, these phases are not aftershocks, as could easily be verified by examining the records obtained at seismological stations having instruments with different characteristics (such as the Weston and Fordham Benioffs). As the reader may verify, ${ }^{27}$ the published lists of aftershocks do not indicate any shocks whose arrival times would fit those of the Airy phase.

We purposely did not include the aftershock of August 7, 1946, $H=$ $19: 21: 32$, in our list, since it was followed by another shock $15 \mathrm{~min}$. $35 \mathrm{sec}$. later which did interfere with the Airy phase of the former.

We see, therefore, that this arrival is clearly a new phase, which cannot be explained by any of the standard types of elastic waves transmitted through the earth's outer or inner layers.

\footnotetext{
${ }^{26}$ Harold Jeffreys and K. E. Bullen, "Seismological Tables," British Assoc. Adv. Sci. (1940).

${ }^{27} \mathrm{New}$ Engl. Seism. Assoc., Bulletin, August, 1946; Bull. Seism. Lab., Calif. Inst. of Tech., July-September, 1946.
} 
TABLE 2

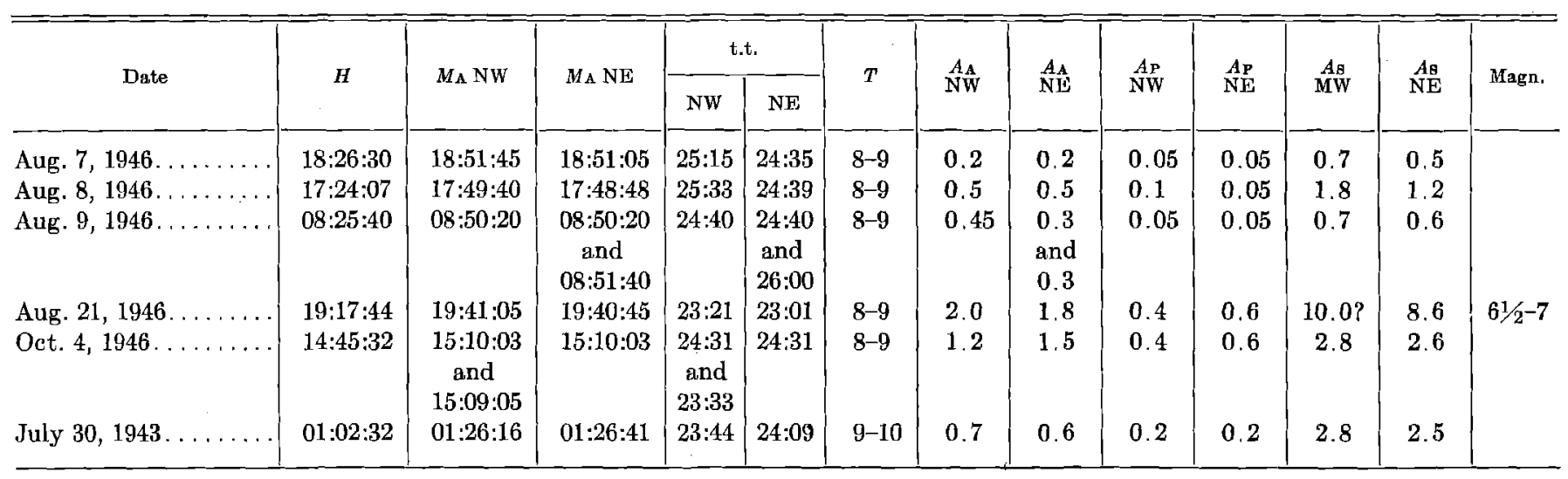


Figure 16, $a, b$, reproduces seismograms for both components of the shocks of August 8, $1946(H=17: 24: 07)$ and August 9, $1946(H=08: 25: 40)$. The phase in question is seen to stand out very conspicuously from the late coda. On all records examined it stands out with the same perfect clarity, and even for shocks much smaller than any of those enumerated it is possible to detect it, though it is not easy to get good times.

In order to obtain the velocity of this phase we should have a fairly accurate idea of the distance from the epicenter. Unfortunately, it is not possible to obtain the accurate readings of the $\mathrm{P}-\mathrm{S}$ interval necessary for this purpose from this type of seismogram. We will have to be content with an approximate evaluation.

The largest of the shocks listed in table 2 is that of October 4, 1946, $H=$ $14: 45: 32$. Its epicenter is given by Gutenberg as $18^{\circ} 45^{\prime} \mathrm{N}, 68^{\circ} 30^{\prime} \mathrm{W}$. This means $\Delta=14^{\circ} 03^{\prime}$. Taking the travel time as $24 \mathrm{~min}$. 31 sec., we get a velocity of $3480 \mathrm{ft} / \mathrm{sec}$, which is 0.7 of the speed of sound in water.

Considering the epicenter to have been determined to within $\pm 1^{\circ}$, which is a reasonable estimate, the velocity given above is accurate to within \pm 250 $\mathrm{ft} / \mathrm{sec}$., i.e., it is somewhere between 0.75 and 0.65 of the velocity of sound in water.

The period of this phase being on the record of the order of 9 sec., it is seen that it is in approximate agreement with the theoretically predicted Airy phase. It might be necessary to assume a higher bottom velocity than that of granite between Bermuda and the West Indies in order to explain its slightly lower velocity.

As for the differences in travel times of the Airy phases of table 2 , it should be remembered that they are sensitive to differences in distance since the velocity of propagation is comparatively low. Thus a difference in distance from the station to the epicenters of two shocks of $1^{\circ}$ would cause a difference in travel times of the order of $1 \mathrm{~min} .40$ sec. This is of the order of magnitude of the differences shown in table 2 .

Bermuda is obviously ideally located for the recording of Airy phases of the Dominican Republic shocks since the entire paths are oceanic. However, a more favorable type of instrumentation would be highly desirable in order to make a thorough study of the Airy phase. In particular, a vertical-component seismometer, with a peak sensitivity in the vicinity of 11 sec., a higher magnification and greater paper speed would be useful in obtaining more accurate data.

These Airy phases do not show up on the Milne-Shaw seismograms of the Dominion Observatory in Ottawa. Probably an appreciable amount of the energy of the phase is lost in the transfer from ocean to continent.

A fair-sized shock occurred on the western flank of the Mid-Atlantic Ridge on May 6, 1944, at $22^{\circ} .4 \mathrm{~N}, 44^{\circ} 8 \mathrm{~W}$ (U.S.C.G.S.), $\mathrm{H}=00: 13.7$. This appears 


(

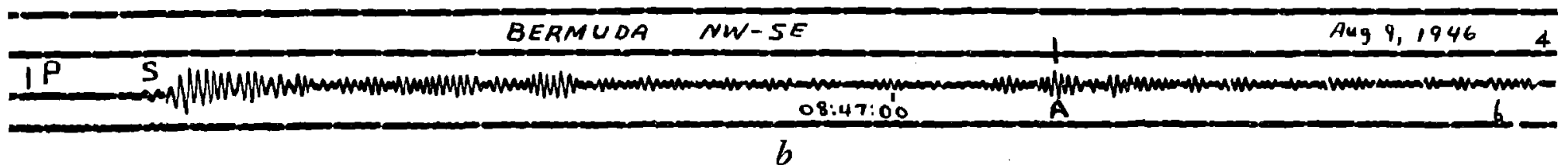

Fig. 16. Airy phase recorded at Bermuda for the earthquakes (a) 1946 August $8^{d} 17^{\text {h }}$ and (b) 1946 August $9^{d} 8^{\text {h }}$. 
to be the only one of the series of Mid-Atlantic Ridge quakes in recent years to have produced a recognizable Airy phase on the Bermuda seismograms. The amplitude of the group of waves interpreted as such is small; nevertheless, this group is fairly well-individualized and stands out well from the rest of the late coda. The period is 9 sec. and the arrival time $00: 46: 27$, which means a travel time of $32 \mathrm{~min} .45 \mathrm{sec}$. for $\Delta=20^{\circ} 17^{\prime}$, i.e., a velocity of $3760 \mathrm{ft} / \mathrm{sec} ., 0.75$ of the velocity of sound in water. The double-trace amplitudes in $\mathrm{cm}$. read as follows. NE: P, 0.25; S, $2.0 ; \mathrm{A}, 0.3 ; \mathrm{NW}: \mathrm{P} ; 0.7 ; \mathrm{S}, 1.3 ; \mathrm{A}, 0.5$.

Huancayo. - The Huancayo Magnetic Observatory is situated at $12^{\circ} 03^{\prime} \mathrm{S}$, $75^{\circ} 20^{\prime} \mathrm{W}$. It is equipped with two Wenner seismographs oriented N-S and $\mathrm{E}-\mathrm{W}$. The natural period is given as 10 sec. and the magnification as 1550 by the U.S.C.G.S. seismological bulletin. It also has a Benioff vertical of period 1 sec. and magnification unknown.

On Aúgust 5, 1944, both components of the Wenner recorded what appears to be the Airy phase of a shock which occurred at $01: 24: 08$ at $131 / 2{ }^{\circ} \mathrm{S}, 921 / 2 \mathrm{~W}$ (U.S.C.G.S.), southwest of the Galapagos Islands. The arrival time was $01: 50: 42$, which gives a travel time of $26 \mathrm{~min}$. $34 \mathrm{sec}$. Since $\Delta=17^{\circ}$ the velocity comes out to be $3,890 \mathrm{ft} / \mathrm{sec}$. or 0.8 of the velocity of sound in water. The period is 8 sec. Figure 17 shows this Airy phase. It is seen to be very conspicuous. The amplitudes in $\mathrm{cm}$. are as follows. $\mathrm{E}-\mathrm{W}: \mathrm{P}, 1.1 ; \mathrm{S}, 1.4 ; \mathrm{A}, 1.0$; $\mathrm{N}-\mathrm{S}: \mathrm{P}, 0.3 ; \mathrm{S}, 2.0 ; \mathrm{A}, 0.7$. It did not record on the Benioff vertical, the period of which is too short. This latter record proves conclusively that this is not a separate shock, but a phase of the quake recorded selectively only on the Wenner.

Discussion.-The velocities obtained for the Airy phase at Bermuda for the October 4, 1946, Dominican Republic and the May 6, 1944, Mid-Atlantic Ridge quakes and at Huancayo for the August 5, 1944, Galapagos shock are respectively $3480 \pm 250 \mathrm{ft} / \mathrm{sec} ., 3,760$ and $3,890 \mathrm{ft} / \mathrm{sec}$.

The second and third shocks were smaller than the first, so that, in spite of the greater paths traveled in each, the estimated velocities cannot be considered as being any more accurate. The difference between the first two velocities, $3,480 \pm 250$ and 3,760 is therefore sufficiently small to be explainable by errors in the epicentral determinations.

As for the velocity obtained from the Galapagos shock, it should be remembered that Huancayo is situated a little more than 150 miles inland, so that the velocity of $3,890 \mathrm{ft} / \mathrm{sec}$. is probably a little high. It appears only reasonable to assume that once the Airy-phase-type motion reaches land it starts traveling with a velocity similar to the velocity of microseisms on land, roughly three times as fast as its velocity along an ocean path. Allowing for the path across land, the velocity of the Airy phase through the ocean for this shock is 0.7 of the velocity of sound in water. There are therefore no real discrepancies in our figures for the velocities. 

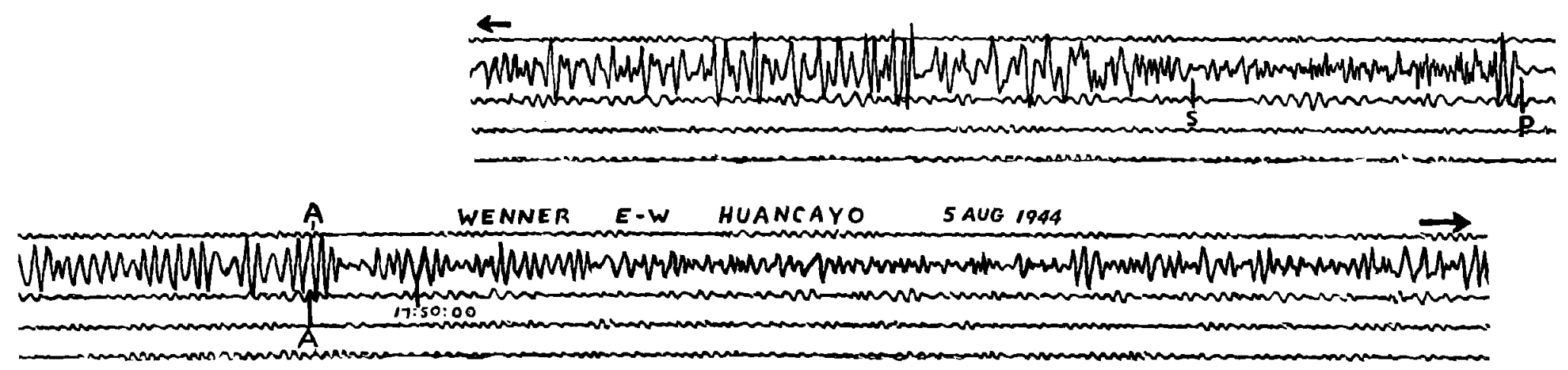

$a$

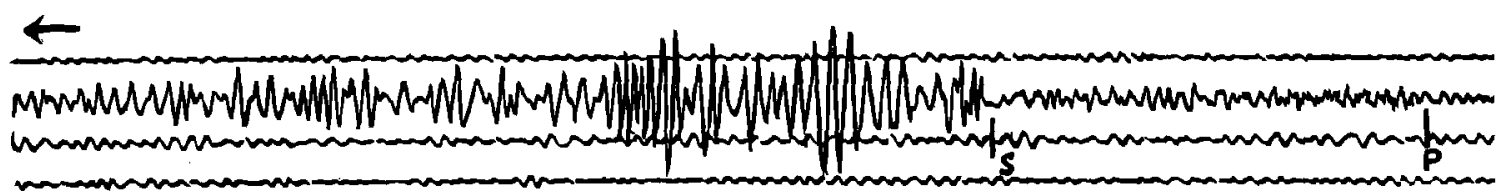

A WENNER N-S HUANCAYO SAUG 1944

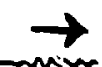

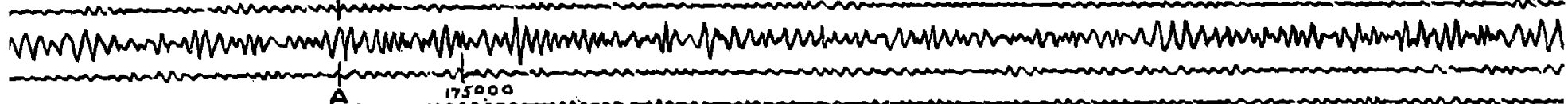

$b$

Fig. 17. Airy phase recorded at Huancayo for the earthquake of 1934 August $5^{\mathrm{d}} 17^{\mathrm{h}}$. 
As for the amplitudes of the various Airy phases, it appears that when the epicenter of the shock is in approximately the same azimuth as one of the components of the recording instrument, it is this component that comes out largest. This is the case for the Mid-Atlantic Ridge earthquake of May 6, 1944 (the $\mathrm{N}-\mathrm{W}$ component of the Airy phase is the largest), and for the Galapagos quake (the $\mathrm{E}-\mathrm{W}$ component is predominant). These two records appear, therefore, to suggest that in the horizontal plane the motion is mostly longitudinal. Because of their orientation the Bermuda records do not yield any information on that score (table 2 shows that the amplitudes are about the same on both components).

We have already mentioned the fact that for the two large shocks of November 25, 1941 (371/2 $\mathrm{N}, 1812^{\circ} \mathrm{W}$, magn. 81/4), and April 11, 1946 (1 ${ }^{\circ} \mathrm{S}$, 141/2 $\mathrm{O}$, magn. 7:2), the Bermuda Milne-Shaws failed to record any Airy phases. A bathymetric chart of the Atlantic Ocean shows that the phases would have been blocked by the Atlantic Ridge. We believe that this provides an adequate explanation of the failure of the Airy phases to show up in the Bermuda instruments. The Mid-Atlantic Ridge forms a continuous barrier across the Atlantic, 100 or more miles in width, rising well above the 2,000-fathom line throughout its whole length, except for one or two narrow trenches. In sections it rises above the 1,000-fathom line. It is to be expected that an Airy phase reaching this barrier would lose much of its energy. What would be left after crossing this barrier would not have enough amplitude to reach Bermuda.

As for shocks occurring on the western flank of the Ridge, all those which have occurred in recent years would have been too small to produce an Airy phase with appreciable amplitude.

It also seems probable that the Airy phase does not propagate over distances of the order of several thousand miles, or at least, if it does, its amplitude is not large enough to distinguish it from the very late coda. Thus the torsion seismometers and linear strain seismometers of the West Coast do not show any Airy phases for the Alaskan and Japanese shocks, and neither do the Honolulu Milne-Shaws; the latter, it is true, are troubled by a very high background.

Mechanism of production of an Airy phase.-It can be shown that an impulse either in the liquid layer or in the bottom will produce waves that will propagate according to the laws of normal mode theory.

Thus any earthquake whose epicenter is at sea or close to the shore will produce, among other types of waves, an Airy phase after propagation for a considerable distance through the acoustic system consisting of ocean and bottom. However, it will be only under certain given conditions that this phase will have energy enough to produce a noticeable effect on a seismometer situated at a certain distance. 
It will be noted that all the shocks for which we have found Airy phases are shallow-focus ones. The focus is therefore situated practically on the liquidsolid interface. The impulse on the liquid layer is strongest under these conditions. It is probable that the Airy phase produced by a shock whose focal depth is appreciably over $5-10 \mathrm{~km}$. is too weak to be recorded at any distance.

All our data refer to records obtained by seismometers situated on land. It seems quite probable that, with the aid of sensitive hydrophones in the ocean, at sufficient depth to remove the effect of surface gravity waves, it would be possible to record the Airy phases of a great many more shocks and at considerably greater distances.

Practical applications.--The study of the Airy phase of a sufficient number of seaquakes would provide interesting information concerning the nature of the ocean bottom, since the latter has an influence upon both the periods and the velocities of the Airy phase.

Conversely, once the velocities of the Airy phase are known for certain paths, the recording of these by means of suitable instruments could be useful for the more accurate determination of epicentral distances and epicenter locations.

\section{ACKNOWLEDGMENTS}

The authors wish to express their thanks to the offices of the U. S. Coast and Geodetic Survey, Section of Seismology, for making the Bermuda records available. Professors B. Gutenberg and C. F. Richter of the California Institute of Technology were extremely helpful during this investigation. We wish to thank Father Daniel Linehan, S.J., of the Weston Seismological Observatory, Dr. F. A. Hodgson of the Dominion Observatory of Canada, Professor Perry Byerly of the University of California, Father Joseph J. Lynch, S.J., and Dr. William A. Lynch of Fordham University for permission to study records of the respective observatories. We are indebted to Marie Flanagan for computing and drafting the graphs presented in this paper.

Lamont Geological Ogservatory

(Coldmbia University)

Palisades, N. Y.

(Lamont Geological Observatory Contribution No. 8) 